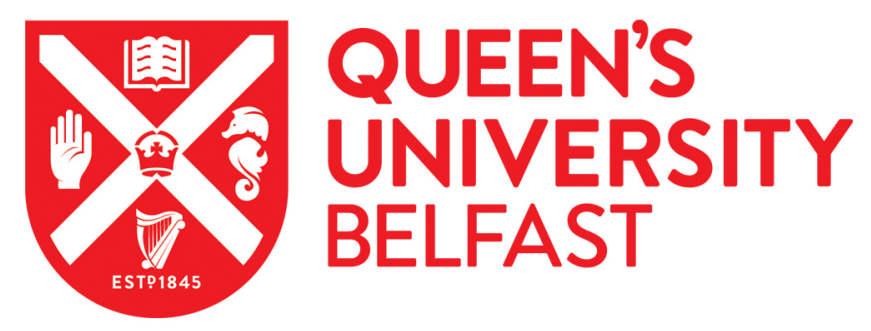

\title{
Signatures of circumstellar interaction in the Type IIL supernova ASASSN-15oz
}

Azalee Bostroem, K., Valenti, S., Horesh, A., Morozova, V., Kuin, N. P. M., Wyatt, S., Jerkstrand, A., Sand, D. J., Lundquist, M., Smith, M., Sullivan, M., Hosseinzadeh, G., Arcavi, I., Callis, E., Cartier, R., Gal-Yam, A., Galbany, L., Gutiérrez, C., Andrew Howell, D., ... Young, D. R. (2019). Signatures of circumstellar interaction in the Type IIL supernova ASASSN-15oz. Monthly Notices of the Royal Astronomical Society, 485(4), 5120-5141. https://doi.org/10.1093/mnras/stz570

Published in:

Monthly Notices of the Royal Astronomical Society

Document Version:

Publisher's PDF, also known as Version of record

Queen's University Belfast - Research Portal:

Link to publication record in Queen's University Belfast Research Portal

Publisher rights

(C) 2019 The Author(s). Published by Oxford University Press on behalf of the Royal Astronomical Society. This work is made available online in accordance with the publisher's policies. Please refer to any applicable terms of use of the publisher.

\section{General rights}

Copyright for the publications made accessible via the Queen's University Belfast Research Portal is retained by the author(s) and / or other copyright owners and it is a condition of accessing these publications that users recognise and abide by the legal requirements associated with these rights.

Take down policy

The Research Portal is Queen's institutional repository that provides access to Queen's research output. Every effort has been made to ensure that content in the Research Portal does not infringe any person's rights, or applicable UK laws. If you discover content in the Research Portal that you believe breaches copyright or violates any law, please contact openaccess@qub.ac.uk. 


\title{
Signatures of circumstellar interaction in the Type IIL supernova ASASSN-15oz
}

\section{K. Azalee Bostroem ${ }^{\odot},{ }^{1 \star}$ Stefano Valenti, ${ }^{1}$ Assaf Horesh, ${ }^{2}$ Viktoriya Morozova, ${ }^{3}$ \\ N. Paul M. Kuin, ${ }^{4}$ Samuel Wyatt, ${ }^{5}$ Anders Jerkstrand, ${ }^{6}$ David J. Sand,${ }^{5}$ Michael Lundquist, ${ }^{5}$ Mathew Smith, ${ }^{7}$ Mark Sullivan, ${ }^{7}$ Griffin Hosseinzadeh, ${ }^{8}$ Iair Arcavi, ${ }^{9}$ Emma Callis ${ }^{\oplus},{ }^{10}$ Régis Cartier, ${ }^{11}$ Avishay Gal-Yam, ${ }^{12}$ Lluís Galbany ${ }^{\oplus}, 13$ Claudia Gutiérrez, ${ }^{7}$ D. Andrew Howell, ${ }^{14,15}$ Cosimo Inserra ${ }^{\bullet},{ }^{16}$ Erkki Kankare, ${ }^{17,18}$ Kristhell Marisol López ${ }^{\odot},{ }^{19,20}$ Curtis McCully, ${ }^{14,15}$ Giuliano Pignata, ${ }^{21,22}$ Anthony L. Piro, ${ }^{23}$ Ósmar Rodríguez ${ }^{\oplus},{ }^{21,22}$ Stephen J. Smartt, ${ }^{17}$ Kenneth W. Smith, ${ }^{17}$ Ofer Yaron ${ }^{12}$ and David R. Young ${ }^{17}$}

Affiliations are listed at the end of the paper

Accepted 2019 February 19. Received 2019 February 14; in original form 2018 November 22

\begin{abstract}
Hydrogen-rich, core-collapse supernovae are typically divided into four classes: IIP, IIL, IIn, and IIb. Recent hydrodynamic modelling shows that circumstellar material is required to produce the early light curves of most IIP/IIL supernovae. In this scenario, IIL supernovae experience large amounts of mass-loss before exploding. We test this hypothesis on ASASSN15oz, a Type IIL supernova. With extensive follow-up in the X-ray, UV, optical, IR, and radio, we present our search for signs of interaction and the mass-loss history indicated by their detection. We find evidence of short-lived intense mass-loss just prior to explosion from light-curve modelling, amounting in $1.5 \mathrm{M}_{\odot}$ of material within $1800 \mathrm{R} \odot$ of the progenitor. We also detect the supernova in the radio, indicating mass-loss rates of $10^{-6}$ to $10^{-7} \mathrm{M}_{\odot} \mathrm{yr}^{-1}$ prior to the extreme mass-loss period. Our failure to detect the supernova in the X-ray and the lack of narrow emission lines in the UV, optical, and NIR do not contradict this picture and place an upper limit on the mass-loss rate outside the extreme period of $<10^{-4} \mathrm{M}_{\odot} \mathrm{yr}^{-1}$. This paper highlights the importance gathering comprehensive data on more Type II supernovae to enable detailed modelling of the progenitor and supernova which can elucidate their mass-loss histories and envelope structures and thus inform stellar evolution models.
\end{abstract}

Key words: techniques: imaging spectroscopy-stars: late-type-supernovae: generalsupernovae: individual: ASASSN-15oz-stars: winds, outflows.

\section{INTRODUCTION}

Stars greater than $8 \mathrm{M}_{\odot}$ end their lives spectacularly as corecollapse supernovae (CCSNe). CCSNe are divided into two types: Type I, those without hydrogen in their spectra and Type II, those with hydrogen in their early spectra (Minkowski 1941). Although the diversity of Type II light curves was noted early (Minkowski 1964; Pskovskii 1967), it was Barbon, Ciatti \& Rosino (1979) who first proposed a division of the Type II class into Type IIP SNe (IIP $\mathrm{SNe}$ ) and Type IIL SNe (IIL SNe) based on the shape of their light

\footnotetext{
*E-mail: abostroem@gmail.com
}

curves. With small samples, some authors have found a distinction between IIP SNe and IIL SNe light curves (e.g. Patat et al. 1993; Patat et al. 1994; Arcavi et al. 2012). However, the recent analysis of large samples of SNe by Anderson et al. (2014), Faran et al. (2014), Sanders et al. (2015), Valenti et al. (2016), Galbany et al. (2016), and Rubin \& Gal-Yam (2016) shows a continuum between the archetypical IIP and IIL light curves. Given the continuum of light curves observed, we will use IIP-like to denote SNe at the IIP end of the spectrum and IIL-like to denote SNe at the IIL end of the spectrum.

From high-resolution imaging prior to the SNe explosion, the progenitors of IIP/IIL SNe are found to be red supergiants (RSGs) with masses $<17 \mathrm{M}_{\odot}$ (Smartt 2015 and references there in). 
Successful modelling of IIP-like SNe with RSG progenitors has shown that the initial period of decline is due to adiabatic cooling during the expansion of the ejecta (e.g. Grassberg, Imshennik \& Nadyozhin 1971; Falk \& Arnett 1977; Blinnikov \& Bartunov 1993; Kasen \& Woosley 2009). When the photosphere reaches $\sim 6000 \mathrm{~K}$, hydrogen recombination commences, moving the photosphere inward, against the outwardly expanding ejecta. The rate of the expansion of the ejecta matches the rate of the recession of the recombination front, which itself is at the constant temperature of recombination, leading to the almost constant brightness that defines the IIP-like class. When the photosphere passes completely through the hydrogen dominated layer of the ejecta, the ejecta continues to expand and cool, as layers rapidly become optically thin until the light curve is powered by the radioactive decay of ${ }^{56} \mathrm{Ni} \rightarrow{ }^{56} \mathrm{Co} \rightarrow{ }^{56} \mathrm{Fe}$.

It is theorized that IIL-like SN light curves arise from progenitors with smaller hydrogen envelopes (Grassberg et al. 1971; Young \& Branch 1989; Blinnikov \& Bartunov 1993). In this scenario, the recombination front would move faster than the expanding ejecta, causing the brightness to decrease. The shallow $\mathrm{H} \alpha$ absorption troughs in IIL-like SNe, possibly due to less absorbing material, support this picture (Schlegel 1996; Gutiérrez et al. 2014). To explode with smaller hydrogen envelopes, IIL-like SN progenitors must lose mass via stellar winds or mass transfer to a binary companion. In this paper we will consider the case of mass-loss via stellar winds. As mass-loss increases with zero-age main-sequence mass (M $M_{\text {ZAMS }}$ ) (Heger et al. 2003; Kasen \& Woosley 2009), this leads to the hypothesis that the progenitors of IIL-like SNe are more massive than IIP-like progenitors. Observations that IIL-like SNe have shorter periods before falling to the radioactive decay phase are brighter, and have higher velocity ejecta support this hypothesis (Patat et al. 1994; Anderson et al. 2014; Faran et al. 2014; Gutiérrez et al. 2014; Sanders et al. 2015; Valenti et al. 2015, 2016). This idea is intriguing as it solves another outstanding problem, the lack of progenitors between 17 and $25 \mathrm{M}_{\odot}$ (Smartt 2015) (for other possible solutions see: Walmswell \& Eldridge 2012; Davies \& Beasor 2018). However, Valenti et al. (2016) found that there is not a clear difference in the progenitor population of IIP-like and IILlike $\mathrm{SNe}$ and more importantly, that the progenitors of IIL-like SNe are not above $17 \mathrm{M}_{\odot}$. Nevertheless, mass-loss, whether governed by progenitor mass or some other factor (e.g. metallicity, rotation, binarity) remains the most promising explanation for the diversity of light-curve shapes.

Mass-loss rates for RSGs are not well understood theoretically and are extremely difficult to characterize with observations due to both the short period of this phase as well as the rarity of high-mass stars. Thus the mass-loss rates for RSG are uncertain, with typical mass-loss rate estimates between $10^{-6}$ and $10^{-4} \mathrm{M}_{\odot} \mathrm{yr}^{-1}$ and wind velocities between 10 and $100 \mathrm{~km} \mathrm{~s}^{-1}$ (Mauron \& Josselin 2011). Mass lost from an SN progenitor over the course of its lifetime will be distributed around the progenitor with a density that is related to the time the mass-loss occurred, the mass-loss rate, and the wind velocity. Although interaction has been observed in $\mathrm{SNe}$ at the edge of the distribution in terms of decline rate (steep) and absolute magnitude (bright; e.g. SN 1979C: Blinnikov \& Bartunov 1993; SN 1980K: Chugai 1992; SN 1998S: Chugai 2001; see Section 7), it is often assumed to be negligible in IIP/IIL SNe. It is possible that the signatures of interaction with circumstellar material (CSM; with stellar wind origins) are visible in the light curves and spectra of most IIP/IIL SNe, especially outside of the optical where there are fewer observations. The detection and evolution of this interaction can directly probe the mass-loss history of massive stars and provide valuable information on the nature of IIP/IIL SN progenitors.

In fact, evidence of interaction, often characterized by a single type of observation (e.g. X-ray detection), indicates that mass-loss should be considered in the interpretation of IIP/IIL SNe. Recent light-curve modelling has shown that dense CSM is required to match the early UV and optical light curves of IIP/IIL SNe with hydrodynamic models of SNe from RSG progenitors (Gezari et al. 2015; Förster et al. 2018; Morozova, Piro \& Valenti 2018; Paxton et al. 2018). Flash ionization features, the narrow emission lines in Type IIP/IIL SNe that disappear within the first hours to days post explosion are also explained by interaction with CSM. These features can be produced either by episodic high-mass-loss rates in single stars (Gal-Yam et al. 2014, e.g. Yaron et al. 2017) or by lower mass-loss rates in a binary system (Kochanek 2019). Additional evidence of the importance of mass-loss comes from rare X-ray and radio observations of IIP/IIL SNe, some of which detect signs of interaction (e.g. Dwarkadas 2014 and references there in, Morozova \& Stone 2018).

Although interaction is seen in some historical IIL-like SNe (e.g. 1979C, 1980K, 1998S), as more IIL-like SNe are discovered, it has become clear that these interacting events are at the edge of the IILlike distribution with bright absolute magnitudes and steep decline rates. Furthermore, a large number of IIL-like SNe, more central to the distribution, lack strong signs of interaction. Here, we search for interaction in one of these central IIL-like SN, discovered by the All-Sky Automated Survey for SuperNovae (ASAS-SN), ASASSN$15 \mathrm{oz}$. With our extensive set of data, ranging from X-ray to radio wavelengths, we examine different indicators of interaction with CSM to form a coherent history of mass-loss.

The outline of the paper is as follows. In Section 2 we describe the data collected at all wavelengths. In Section 3 we present the parameters of ASASSN-15oz. The photometric and spectroscopic evolution are described in Sections 4 and 5, respectively. Section 6 describes our search for interaction, including hydrodynamic lightcurve modelling and radio analysis. Finally, we present a comparison to other objects and a unified interpretation of the mass-loss history indicated by the multiwavelength observations in Section 7 and summarize our conclusions in Section 8.

\section{OBSERVATIONS AND DATA REDUCTION}

ASASSN-15oz was the closest Type II SN of 2015 and was observed extensively at all wavelengths. The majority of our observations are provided by the Las Cumbres Observatory (LCO; Brown et al. 2013) Supernova Key Project (2014 to 2017) and the Public ESO Spectroscopic Survey for Transient Objects (PESSTO). We supplemented these with observations in the X-ray, ultraviolet (UV), optical, near infra-red (NIR), and radio. The photometric and spectroscopic data reduction is described in this section. A table of spectroscopic observations is presented in Table A1. A complete list of photometric observations is given in the Supplemental Material. Table A2 gives an example of the photometric observation table format. All photometric data can be obtained from the SNDAVIS database $^{1}$ (Stefano Valenti) and all spectroscopic data can be found on WISeREP (Yaron \& Gal-Yam 2012). ${ }^{2}$

\footnotetext{
${ }^{1}$ http://dark.physics.ucdavis.edu/sndavis/transient

${ }^{2} \mathrm{https} / / /$ wiserep.weizmann.ac.il
} 


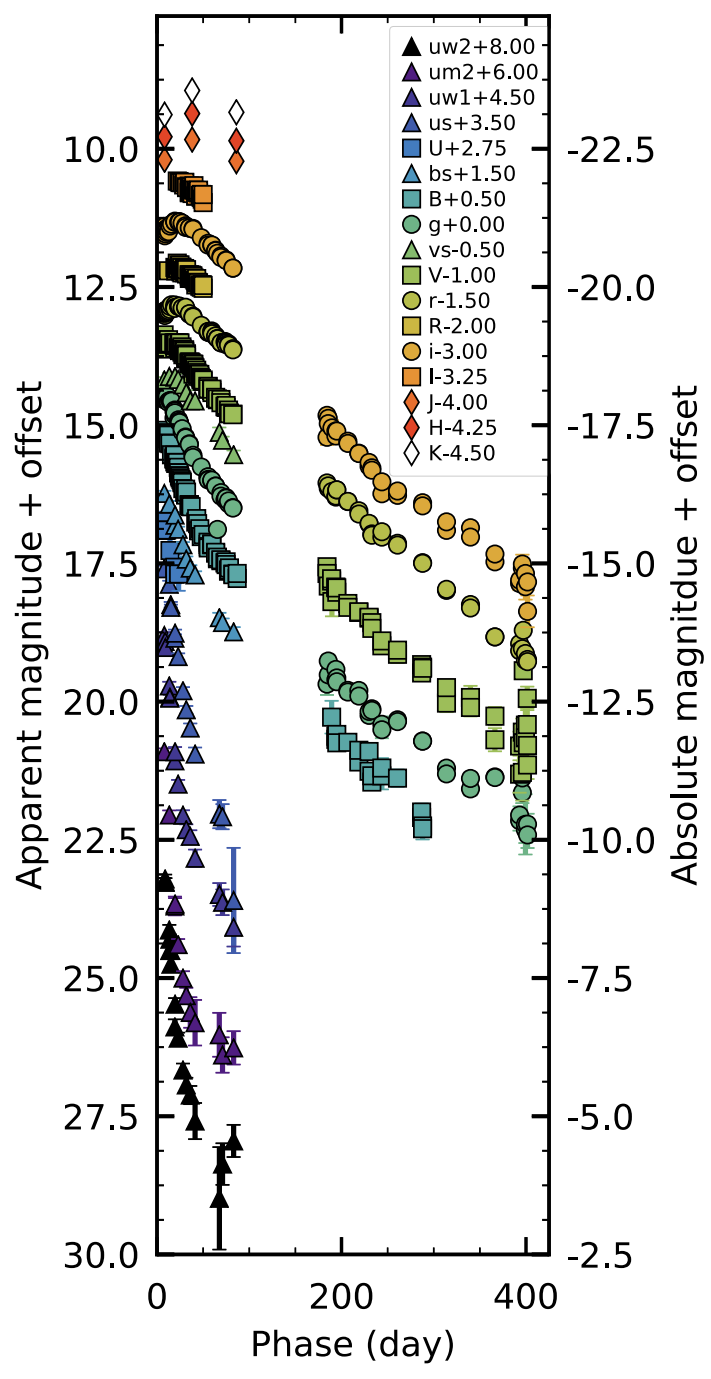

Figure 1. The complete UV, optical, and NIR light curve of ASASSN-15oz expressed in terms of days since explosion. Apparent magnitude (+offset) is plotted on the left and absolute magnitude (+offset) is plotted on the right. Each colour corresponds to a single filter, labelled in the legend. The optical Swift filters are denoted with an 's' in the name (e.g. us) and plotted with the triangle symbol. NIR observations are shown with the diamond symbol. To differentiate the $U, B, V, R$, and $I$ filters and the $g, r$, and $i$ filters, we use a square symbol for the former and a circle for the later. Each filter has been shifted by a global offset (denoted in the legend) for viewing purposes.

\subsection{Optical}

The optical light curve was closely monitored by the LCO from discovery through day 400 in the filters $B, V, g, r$, and $i$. Unfortunately, the SN passed behind the sun around day 80 , leaving a gap in the observations from day 87 to day 179 , most notably missing the fall from plateau. Fig. 1 shows the complete multiband light curve. LCO observations were reduced with the LCO imaging pipeline, lcogtsnpipe (Valenti et al. 2016). This pipeline employs PSF photometry, removing background contamination by fitting a loworder polynomial to the host galaxy. Instrumental magnitudes were converted to apparent magnitudes using stars in the APASS,${ }^{3}$ Sloan, and Landolt catalogues. Apparent magnitudes for the $U, B, V, R$, and
$I$ filters are given in the Vega magnitude system and apparent magnitudes for the $g, r$, and $i$ filters are given in the AB magnitude system.

Extensive optical spectroscopy was obtained during the photospheric phase with the FLOYDS spectrograph (Brown et al. 2013) on the 2-m LCO telescope at Siding Springs, Australia and the EFOSC2 spectrograph (Buzzoni et al. 1984) on the $3.6 \mathrm{~m}$ NTT telescope at La Silla, Chile during the photospheric and nebular phase. One-dimensional spectra were extracted and calibrated using the FLOYDS pipeline (Valenti et al. 2014) and the EFOSC tasks in the PESSTO pipeline (Smartt et al. 2015). Both of these pipelines combine IRAF tasks to bias subtract and flat-field the data and locate, extract, wavelength calibrate, and flux calibrate the one-dimensional spectrum.

Data were also taken using the X-Shooter echelle spectrograph (Vernet et al. 2011) on the 8.2-m Very Large Telescope (VLT) at the European Southern Observatory (ESO). X-Shooter has three arms (UVB, VIS, NIR) which combined provide continuous wavelength coverage over 3000 to $24800 \AA$. For our data, we used slit widths of 1.0, 0.9, and 0.9 arcsec in the UVB, VIS, and NIR arms, corresponding to resolutions of $R \sim 5400,8900$, and 5600, respectively. The UVB and VIS data were reduced via a modified version of the EsoReflex pipeline (Freudling et al. 2013), with improvements to the sky-subtraction and rebinning procedure on the highly dispersed echelle spectrum.

Fig. 2 shows the evolution of the optical spectra over time. The progression starts with a blue spectrum with broad hydrogen lines at the top of the figure. Over time (moving down the figure), the spectra develop metal features such as iron and scandium as the velocities decrease and the line widths narrow.

We obtained late-time spectra from the Gemini South Observatory through program GS-2016A-Q-75-25 (PI: Valenti). The Gemini spectra of ASASSN-15oz were taken on day 287 (2016 June 09), day 288 (2016 June 10), and day 290 (2016 June 12) using the Gemini Multi-Object Spectrograph (GMOS) (Hook et al. 2004). Data were taken with a blue setting with the B600 grating, no filter, a 1.0 in. slit, and $2 \times 2$ binning, and a red setting with the R400 grating, OG515 order blocking filter, a 1.0 in. slit, and $2 \times 2$ binning. These settings provide a wavelength range 3630 to 6830 $\AA$ with a dispersion of $1.0 \AA$ pixel $^{-1}$ in the blue and a wavelength range 5030 to $9800 \AA$ with a dispersion of $1.3 \AA \mathrm{pixel}^{-1}$ in the red. We used the GEMINI IRAF package for GMOS to process our data. The raw two-dimensional images were bias subtracted, overscan corrected, flat-fielded, wavelength calibrated using $\mathrm{CuAr}$ emission lamps, cleaned of cosmic rays, and background subtracted prior to extraction of the 1D target spectra. The spectra were then calibrated using a sensitivity function derived from a standard star observed for the program.

\subsection{UV and X-ray observations}

UV and X-ray imaging observations were obtained concurrently with Swift from day nine (2015 September 05) through day 83 (2015 November 18). In addition to the imaging observations, two epochs were observed with the Swift grism under program 1114241 (PI: Valenti). Imaging observations were processed with aperture photometry following Brown et al. (2009) and employing the updated zero-points of Breeveld et al. (2010). UV grism observations were taken at two roll angles and extracted using the UVOTPY pipeline ${ }^{4}$ (Kuin et al. 2015). There is a nearby star coincident with the SN 


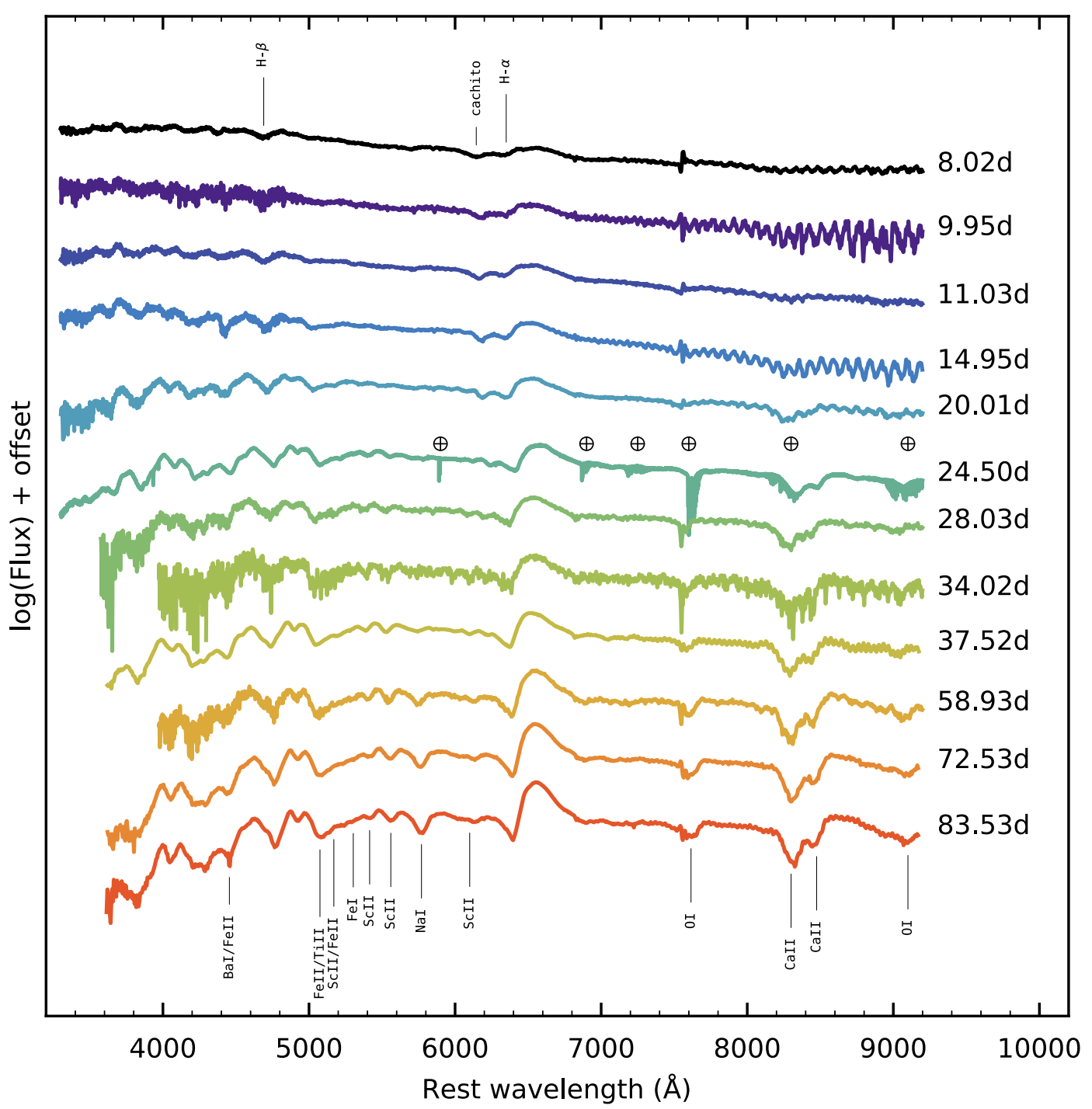

Figure 2. A time series of the photospheric spectra of ASASSN-15oz at rest wavelengths. The phase of each spectrum is marked on the right. Potential telluric contamination is marked with crossed circles above the day 24.5 spectrum. The first spectrum taken near maximum light shows hydrogen features (identified at the top of the figure). At this early phase, the cachito feature (see Section 6.2) is already visible. Over time the cachito feature fades while the hydrogen emission grows and metal lines become visible and grow in strength. These lines are identified in the bottom spectrum.

at one roll angle and just above it at the other roll angle. Although we tried custom extractions using different extraction box height and template subtraction using TRUVOT (Smitka 2015) we were unable to produce a better calibration than the default pipeline. Due to the distance of the ASASSN-15oz, only the first of the two spectroscopic epochs has sufficient $\mathrm{S} / \mathrm{N}$ to be extracted. These spectra are shown in Fig. 3.

$\mathrm{X}$-ray upper limits were calculated using the SOSTA command in the HEASOFT package XIMAGE. Count rates were converted to flux units using PIMMS (Mukai 1993) assuming a power-law model with a photon index of two for an input and output energy range of 0.5 to $8 \mathrm{keV}$. There were no X-ray detections at any epoch.

\subsection{NIR observations}

ASASSN-15oz was observed in the NIR with the SOFI instrument on the NTT telescope through the PESSTO collaboration, the SpeX instrument (Rayner et al. 2003) on the 3.0-m NASA
Infrared Telescope Facility (IRTF), and the NIR arm of the XShooter spectrograph. The data reduction of the NIR arm of the $\mathrm{X}$-Shooter spectrum is described with the UV and VIS arms in Section 2.1.

The SOFI spectroscopic data were reduced with the PESSTO package (Smartt et al. 2015). Photometric measurements were performed with the QUBA pipeline (Valenti et al. 2011), which performs DAOPHOT-based (Stetson 1987) point-spread-function fitting photometry on the SN and on the selected reference stars. $J, H$, and $K$ photometry was then calibrated to the 2MASS magnitude system.

The SpeX data were taken in so-called SXD mode, where the spectrum is cross-dispersed to obtain wavelength coverage from $\sim 0.8$ to $2.4 \mu \mathrm{m}$ in a single exposure, spread over six orders. All observations were taken with the slit aligned along the parallactic angle, and we employed a classic ABBA technique for improved sky subtraction. HD 177074, an A0V star, was observed adjacent in time to the science observations for flux and telluric calibration. The spectrum was reduced in a standard way using the publicly 


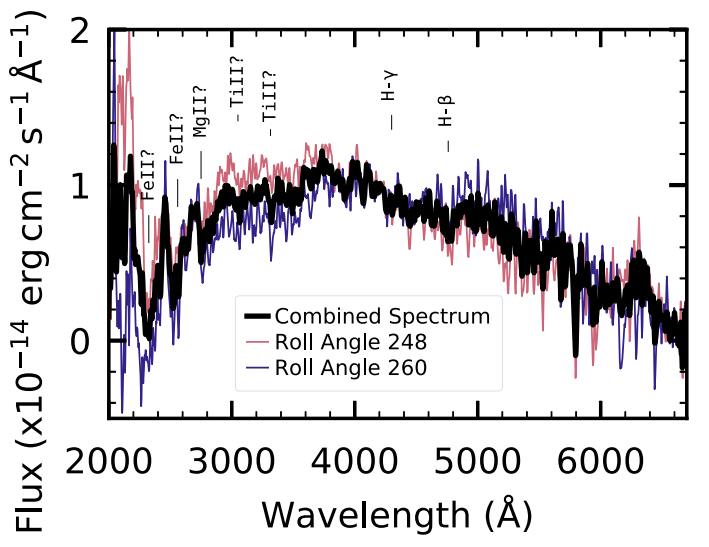

Figure 3. The Swift UV spectra from day nine (2015 September 05), boxcar smoothed with a kernel of three pixels. The spectrum taken with a roll angle of $\mathrm{PA}=248^{\circ}$ is in pink, the spectrum taken with a roll angle of $\mathrm{PA}=260^{\circ}$ in indigo, and average flux is in black. We tentatively identify UV Fe II, Ti II, and $\mathrm{Mg}$ II features as well as mark the Balmer lines. These spectra are not template subtracted, therefore they show residuals from zeroth order light. These features can be identified as being in the spectrum of one roll angle but not the other. The lack of narrow line emission indicates a lack of strong interaction.

available SPEXTOOL software package (Cushing, Vacca \& Rayner 2004) and corrections for telluric absorption utilized XTELLCOR (Vacca, Cushing \& Rayner 2003) and the A0V star observations.

The three photometric observations from day 8 (2015 September 04), day 38 (2015 October 04), and day 86 (2015 November 21) are shown in Fig. 1. Spectroscopic observations from SOFI (day 9; 2015 September 05 and day 39; 2015 October 05), X-Shooter (day 26; 2015 September 21), and SpeX (day 44; 2015 October 10) are shown in Fig. 4.

\subsection{Radio observations}

We observed ASASSN-15oz using the NSF's Karl G. Jansky Very Large Array (VLA) (VLA/15B-362, PI: Valenti) on day 28 (2015 September 24) and day 42 (2015 October 08). In both of the observations we used J1924-2914 and 3C 48 as a phase calibrator and a flux calibrator, respectively. We used the Common Astronomy Software Applications (CASA) standard packages and pipelines to perform the data calibration and imaging. The $\mathrm{SN}$ was detected in both observation with the following flux measurements: September 24: $120 \pm 23 \mu \mathrm{Jy}$ at $4.8 \mathrm{GHz}$ ( $C$ band) and a $3 \sigma$ non-detection limit at $22 \mathrm{GHz}$ ( $K$ band); October $08: 210 \pm 21 \mu \mathrm{Jy}$ at $4.8 \mathrm{GHz}$ ( $C$ band) and an $80 \mu \mathrm{Jy}$ at $15 \mathrm{GHz}$ ( $K U$ band).

\section{SUPERNOVA PARAMETERS}

ASASSN-15oz was discovered by the ASAS-SN team on 2015 August 31.09 (JD 2457 265.59; $V=14.6$ mag; RA = 19:19:33.55, Dec. $=-33: 46: 01.2$ ) and announced on 2015 September 03 (JD 2457 268; Brown et al. 2015). The next day, 2015 September 04 (JD 2457270.0 ), LCO classified it as a Type II SN 1 week after explosion (Hosseinzadeh et al. 2015). Fig. 5 shows the Digital Sky Survey (DSS) image of the low-luminosity host galaxy, HIPASS J1919-33, prior to explosion (top panel) and our first $V$-band image of ASASSN-15oz taken on day 8 (2015 September 04). Imaging of the host galaxy, HIPASS J1919-33, from 2015 August 23 (JD 2457257.56 ) does not show any evidence of ASASSN-15oz $(V>17.8$; Brown et al. 2015). We take this date to be a lower limit on the explosion epoch and define the mid-point between the nondetection and the detection as explosion date: $t_{\text {expl }}=2015$ August $27 \pm 4 \mathrm{~d}$ (JD 2457261.5 ). This is in agreement with the value used in Gutiérrez et al. (2018).

When non-detection observations are not available, other methods can be used to determine the explosion epoch. Gutiérrez et al. (2017) found that the explosion epoch can be determined, with an average errors of $4.9 \mathrm{~d}$, by fitting the blue part of the first spectrum with SNID ${ }^{5}$ (Blondin \& Tonry 2007) and using the explosion epoch of the best fit template. This method relies on the existence of a template SN that is similar in both phase and type to the object being fit. ASASSN-15oz is a IIL-like SN. SNID has very few templates for IIL-like SNe, even after adding the new templates provided by Gutiérrez et al. (2017). Therefore, in addition to the Gutiérrez et al. (2017) templates, we add templates for two well observed supernovae with well-constrained explosion epochs: SN 2012A (Tomasella et al. 2013), and SN 2013ej (Valenti et al. 2014; Smartt et al. 2015; Childress et al. 2016; Dhungana et al. 2016). ${ }^{6}$ With these templates, we confirm our explosion epoch by fitting the first spectrum (2015 September 04) using SNID v. 5.0. The five best fit spectra imply an explosion epoch of 2015 August $22 \pm 4.9$ (JD 2457256.5 ), consistent with the range we propose based on non-detections. Interestingly, the hydrodynamic light curve modelling also places the best fit explosion epoch earlier than 2016 August 27 (see Section 4). As the uncertainties of both these methods are greater than that of the non-detection, throughout this paper we adopt the value determined by the non-detection as the explosion epoch, $t_{\text {expl }}=2015$ August $27 \pm 4$ d (JD 2457 261.5), unless otherwise specified.

We use the Hubble flow distance modulus to the host galaxy, HIPASS J1919-33, $\mu=32.3 \pm 0.2 \mathrm{mag}$, with $\mathrm{H}_{0}=73 \mathrm{~km} \mathrm{~s}^{-1}$ $\mathrm{Mpc}^{-1}$ corrected for Virgo infall. ${ }^{7}$ We use the VIS arm of the Xshooter spectrum $(R \sim 8900)$ to constrain the host galaxy reddening and to confirm the galactic reddening using the equivalent widths of the Na I D1 and D2 lines $(\lambda 5890, \lambda 5896)$ (Poznanski, Prochaska \& Bloom 2012). We find no evidence of Na I absorption at the host redshift and therefore assume no host reddening (see Fig. 6). By fitting Gaussian profiles to the galactic NaI absorption we find $E(B-V)=0.12 \pm 0.07 \mathrm{mag}$, consistent with the value given by Schlafly \& Finkbeiner (2011). We adopt the Schlafly \& Finkbeiner (2011) value of $E(B-V)=0.08$ mag for the analysis in this paper and use the extinction law of Cardelli, Clayton \& Mathis (1989) with $R_{\mathrm{V}}=3.1$.

\section{LIGHT-CURVE EVOLUTION}

The light curve of ASASSN-15oz reaches peak brightness in the $V$-band $8.25 \mathrm{~d}$ after explosion, $1 \mathrm{~d}$ after the start of our observations, reaching an above average absolute magnitude of $-18.05 \pm 0.025$ mag. The early light curve rises until around day 20 in the redder filters, flattening in the $V$-band and falling in the blue bands during this same period. It then falls in all bands for the remainder of the observations, changing slope around day 40 and again sometime after day 85 , as it enters the radioactive decay phase, following the

\footnotetext{
${ }^{5}$ Blondin \& Tonry (2011).

${ }^{6}$ SNID templates for SN 2012A and SN 2013ej can be found at https: //github.com/abostroem/snid_templates

${ }^{7}$ The NASA/IPAC Extragalactic Database (NED) is operated by the Jet Propulsion Laboratory, California Institute of Technology, under contract with the National Aeronautics and Space Administration.
} 


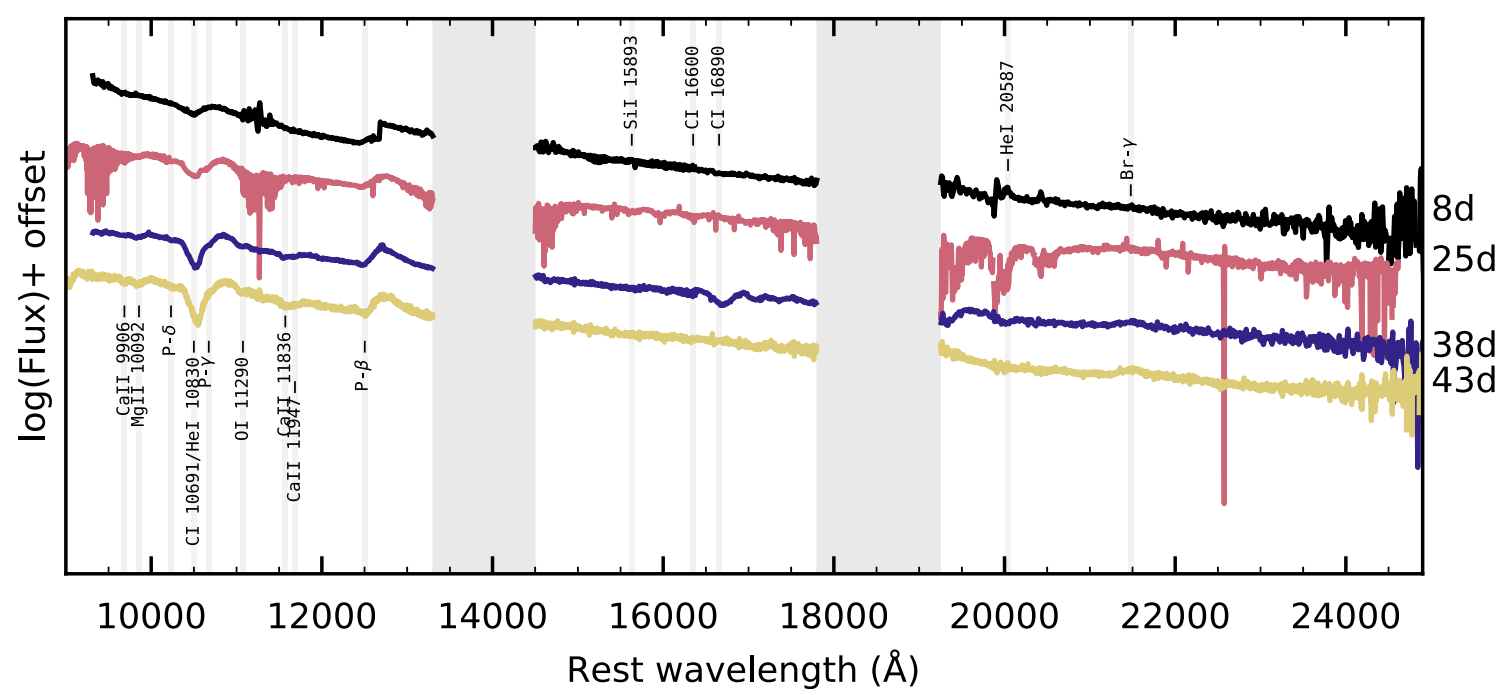

Figure 4. The evolution of the infrared spectra at rest wavelengths during the photospheric phase. The spectral evolution in the IR mirrors the optical evolution, with broad hydrogen features narrowing and metal lines developing over time. The first spectrum (black) taken $8 \mathrm{~d}$ post-explosion shows broad hydrogen features. The later spectra at day 38 (indigo) and 43 (yellow) reveal the development metal lines. Although contaminated with narrow telluric features, the high-resolution X-Shooter spectrum (pink) shows an intermediate phase. Regions of severe sky contamination are marked with grey boxes and are masked out of the spectra.
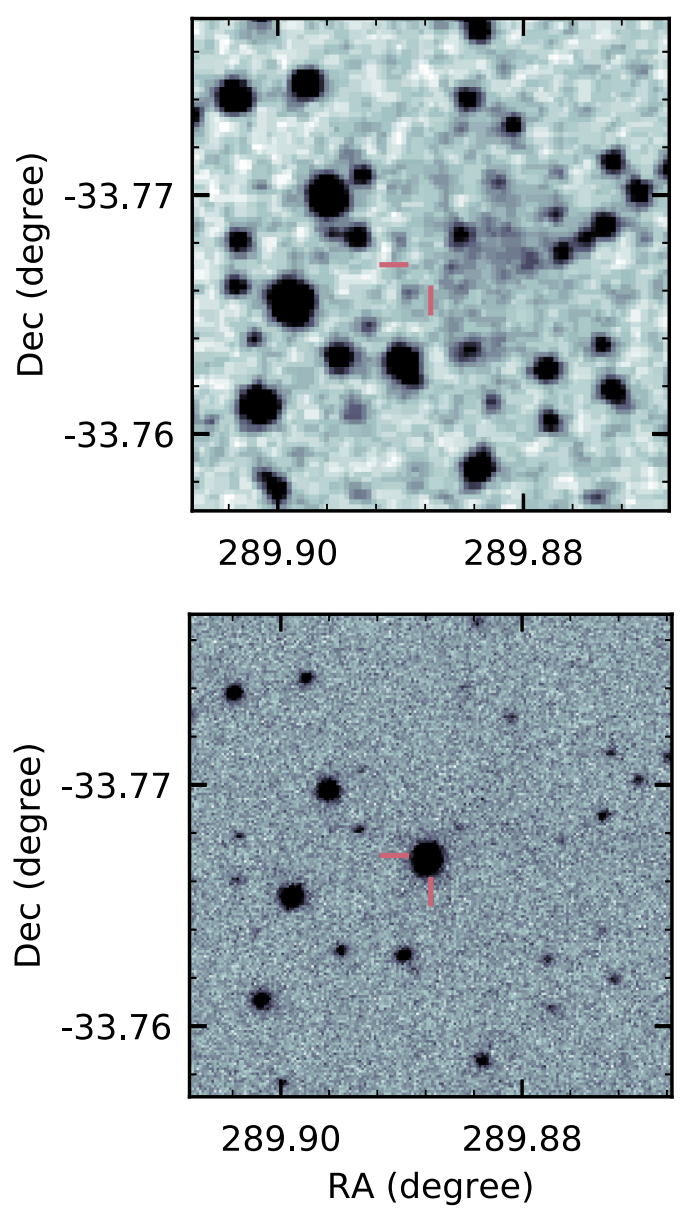

Figure 5. Top: The host galaxy of ASASSN-15oz, HIPASS J1919-33, taken prior to the SN explosion by DSS. Bottom: $V$-band image of ASASSN$15 \mathrm{oz}$ taken by LCO $8 \mathrm{~d}$ post explosion (2015 September 04). The location of the ASASSN-15oz is marked by the pink ticks in both images.

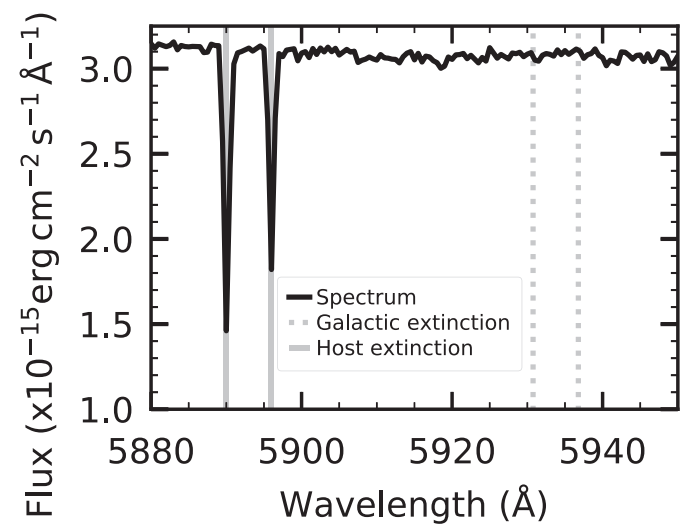

Figure 6. The galactic (solid grey lines) and host (dashed grey lines) Na I $\mathrm{D} 1$ and D2 lines in the high-resolution X-shooter spectrum from day 26 (2015 September 21). The galactic profiles are consistent with the galactic extinction of Schlafly \& Finkbeiner (2011) while the absence of absorption at the host redshift indicates a lack of dust in the host galaxy.

fall from plateau. Following Anderson et al. (2014), we use the $V$-band light curve to fit a slope to the first steep initial decline after maximum $\left(s_{1}\right)$ and to the second shallower slope prior to the fall from plateau $\left(s_{2}\right)$. We also fit a global decline rate, $s_{50 \mathrm{~V}}$ from soon after maximum to prior to the fall from plateau. During the nebular phase we fit a slope to the radioactive decay tail $\left(s_{\text {tail }}{ }^{8}\right)$. The slopes we fit as well as the details of the fit are summarized in Table 1 and plotted on top of the $V$-band light curve in Fig. 7. Both the high slopes and bright maximum luminosity of ASASSN-15oz are similar to those of other IIL-like SNe. The slope of the radioactive decay tail is steeper than expected from the radioactive decay of ${ }^{56} \mathrm{Ni} \rightarrow{ }^{56} \mathrm{Co} \rightarrow{ }^{56} \mathrm{Fe}$ implying that the ejecta is not fully trapping and reprocessing the gamma rays produced by the radioactive decay.

\footnotetext{
${ }^{8}$ This is equivalent to $s_{3}$ in Anderson et al. (2014).
} 
Table 1. The best-fitting slope to the $V$-band light curve of ASASSN-15oz measured between the start and end phase listed in the table. All slopes are measured in units of magnitudes per $50 \mathrm{~d}$.

\begin{tabular}{lcccc}
\hline Slope type & Slope $\left[\mathrm{mag}(50 \mathrm{~d})^{-1}\right]$ & Slope error $\left[\mathrm{mag}(50 \mathrm{~d})^{-1}\right]$ & Start phase $(\mathrm{d})$ & End phase $(\mathrm{d})$ \\
\hline$s_{1}$ & 1.21 & 0.066 & 21.0 & 38.0 \\
$s_{2}$ & 0.99 & 0.021 & 45.0 & 78.0 \\
$s_{50 \mathrm{~V}}$ & 1.09 & 0.014 & 18.4 & 78.0 \\
$s_{\text {tail }}$ & 0.70 & 0.029 & 206.9 & 368.0 \\
\hline
\end{tabular}

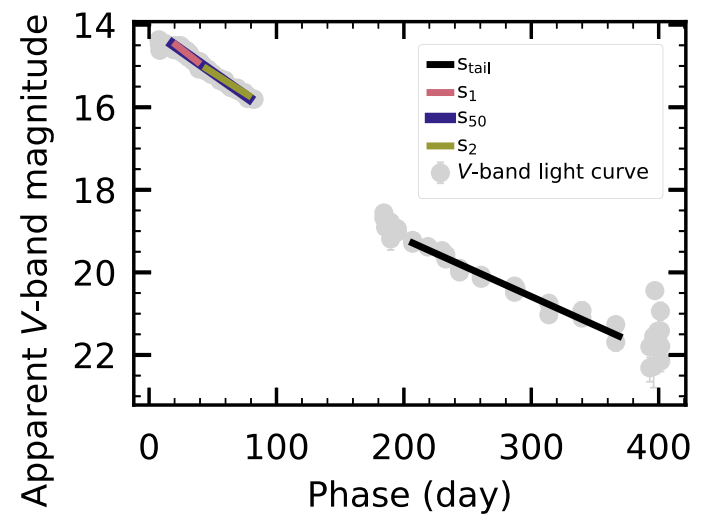

Figure 7. The $s_{1}$ (pink), $s_{2}$ (yellow), $s_{50 \mathrm{~V}}$ (indigo), and $s_{\text {tail }}$ (black) fits plotted over the period used to derive the relation. The $V$-band light curve is plotted in grey. Although errors in the apparent magnitude are plotted, in many cases these are smaller than the points.

The pseudo-bolometric luminosity is computed following Valenti et al. (2008). To summarize the method, the apparent magnitudes are corrected for galactic extinction. We use the $U, B, g, V, r, R$, $i$, and $I$ bands to calculate the pseudo-bolometric luminosity at the $V$-band cadence. The flux density is calculated from the apparent magnitude at the effective wavelength of each filter and integrated using Simpson's Rule. Finally, the flux is converted to a luminosity using the distance modulus. When $V$-band observations extended either earlier or later than a filter, we use the colour with the neighbouring filter to extrapolate the flux.

\subsection{Nickel mass determination}

The amount of ${ }^{56} \mathrm{Ni}$ synthesized during the explosion can be inferred using the late-time ( $>150 \mathrm{~d}$ ) photometry. At this time, the energy output is powered by the radioactive decay of ${ }^{56} \mathrm{Ni} \rightarrow{ }^{56} \mathrm{Co} \rightarrow{ }^{56} \mathrm{Fe}$ which produces gamma rays that are reprocessed by the ejecta producing optical light. Assuming that the SN has the same SED as the extremely well-characterized SN 1987A, the ${ }^{56} \mathrm{Ni}$ mass synthesized in the SN explosion can be found by scaling the pseudobolometric luminosity of $\mathrm{SN}$ when it is powered by ${ }^{56} \mathrm{Co}$ to that of SN 1987A with the following equation (Spiro et al. 2014):

$M\left({ }^{56} \mathrm{Ni}\right)=0.075 \mathrm{M}_{\odot} \times \frac{L_{\mathrm{SN}}(t)}{L_{87 \mathrm{~A}}(t)}$,

where $M\left({ }^{56} \mathrm{Ni}\right)$ is the synthesized ${ }^{56} \mathrm{Ni}$ mass, $L_{S N}(t)$ is the pseudobolometric luminosity of the $\mathrm{SN}$ at time $t$, and $L_{87 \mathrm{~A}}(t)$ is the pseudo-bolometric luminosity of SN 1987A at time $t$ using the same filters as were used to compute the pseudo-bolometric luminosity of the SN.

This scaling can be performed at any time, as long as the slope is the same as that of SN1987A, i.e. consistent with the complete

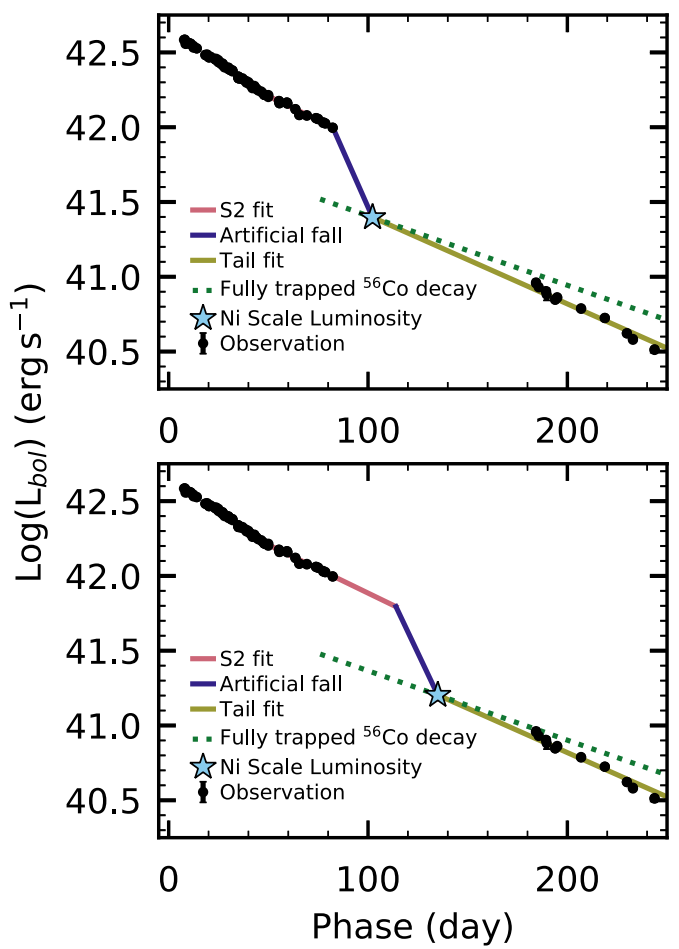

Figure 8. The artificial light curve of ASASSN-15oz for the shortest (top) and longest (bottom) plateau lengths. The black points plot the bolometric luminosity while the pink line represents a fit to the $s_{2}$ data, the indigo line is the artificial fall from plateau, and the yellow line is a fit to the radioactive decay tail. The cyan star marks the point to which the light curve of SN 1987A is scaled to derive a ${ }^{56} \mathrm{Ni}$ mass. Plotted as a green dashed line is the slope of the radioactive decay tail if there was complete trapping. An upper limit on the ${ }^{56} \mathrm{Ni}$ mass of $0.11 \mathrm{M}_{\odot}$ is derived from the light curve in the top panel while a lower limit of $0.08 \mathrm{M}_{\odot}$ is derived from the lower panel.

trapping of gamma rays. As the SN ages, the optical depth of the gamma rays decreases. Thus an $\mathrm{SN}$ is most likely to have complete trapping immediately after the fall from plateau. Unfortunately, for ASASSN-15oz, there is not complete trapping for any part of the observed radioactive decay tail and there are no observations of the period immediately after the fall from plateau. We therefore consider the maximum and minimum ${ }^{56} \mathrm{Ni}$ mass allowed by the observations, under the assumption that there is complete trapping immediately following the fall from plateau. For both cases we also assume that the fall from plateau takes $20 \mathrm{~d}$, based on light curve fits presented in Valenti et al. (2016). An upper limit on the ${ }^{56} \mathrm{Ni}$ mass is obtained by assuming ASASSN-15oz fell from plateau immediately after the last photospheric point (see upper panel of Fig. 8). Scaling the pseudo-bolometric luminosity of SN1987A at $102 \mathrm{~d}$ to that of ASASSN- $15 \mathrm{oz}$ at the same time, we calculate a ${ }^{56} \mathrm{Ni}$ mass of $0.11 \mathrm{M}_{\odot}$. To find the lower limit, we estimate a conservative value 


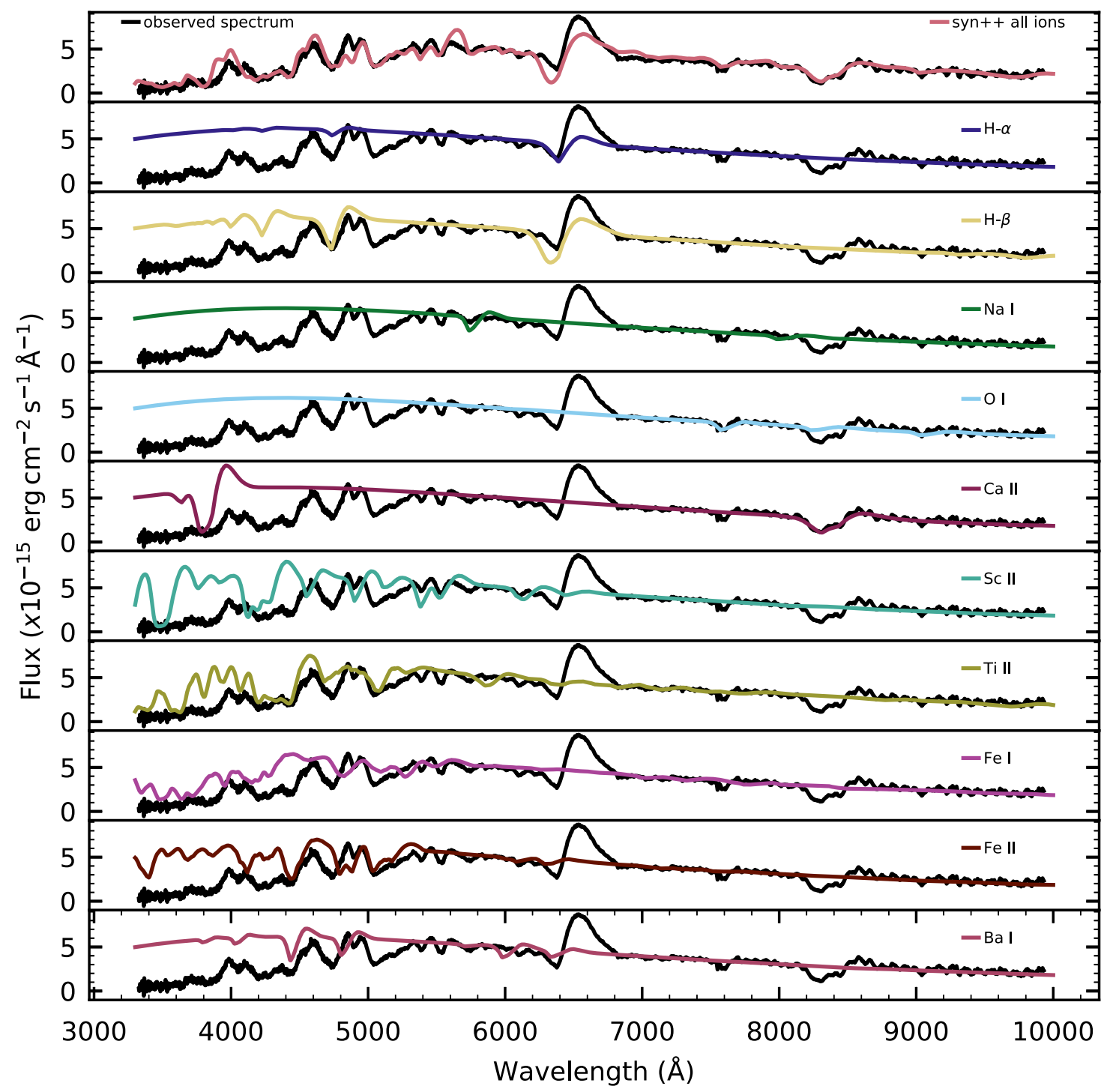

Figure 9. A comparison of the model spectra produced by SYN++ (coloured lines) and the observed spectrum (black) from day 40 (2015 October 06). The top panel shows the best fit with all elements while the remaining panels show the fit for each element individually. We fit $\mathrm{H} \alpha$ and $\mathrm{H} \beta$ separately as $\mathrm{H} \alpha$ is not well modelled by the pure resonance scattering assumed in $\mathrm{SYN}++$. The $\mathrm{H} \beta$ fit is used in the combined spectrum in the top panel.

of the time to the middle of the fall from plateau $\left(t_{P T}\right)$ to be $125 \mathrm{~d}$ using fig. 5(a) in Valenti et al. (2016). Using this $t_{P T}$ we calculate the fall from plateau to end $135 \mathrm{~d}$ after explosion and extrapolate the tail fit to this phase (see lower panel of Fig. 8). We compute a ${ }^{56} \mathrm{Ni}$ mass of $0.08 \mathrm{M}_{\odot}$ by scaling the pseudo-bolometric luminosities at $135 \mathrm{~d}$. In both panels of Fig. 8, the observations are represented with black circles, the $s_{2}$ slope with a pink line, the fall from plateau with a indigo line, the tail slope with a yellow line, and the expected tail slope from complete trapping is marked with a dashed green line. The time that is used to scale SN 1987A to ASASSN-15oz is marked with a cyan star.

\section{SPECTROSCOPIC ANALYSIS}

The spectroscopic evolution of an $\mathrm{SN}$ during the photospheric phase provides insight into details of the $\mathrm{SN}$ ejecta. The changes in velocity and the individual line profiles describe the geometry and energetics of the ejecta. The presence of different species at different times gives information about the chemical composition, temperature, and density of the ejecta. In this section we analyse optical and NIR spectra.

\subsection{Optical evolution}

Initial line identification was performed by comparing the spectra to that of SN 1999em (Leonard et al. 2002). Identification was confirmed using the spectrum synthesis code SYN $++{ }^{9}$ (Thomas, Nugent \& Meza 2011; parameter details in Table B1). The results of the SYN++ fit can be seen in Fig. 9.

We use two methods to find the velocity of lines for which individual components can be resolved, one for blended features, and one for individual features. For lines that are not blended or that are dominated by the absorption of one ion (e.g. Sc II $\lambda \lambda 5526$, 5662,6262 ), we employ the method presented in Silverman, Kong \&

\footnotetext{
${ }^{9}$ Thomas (2013).
} 


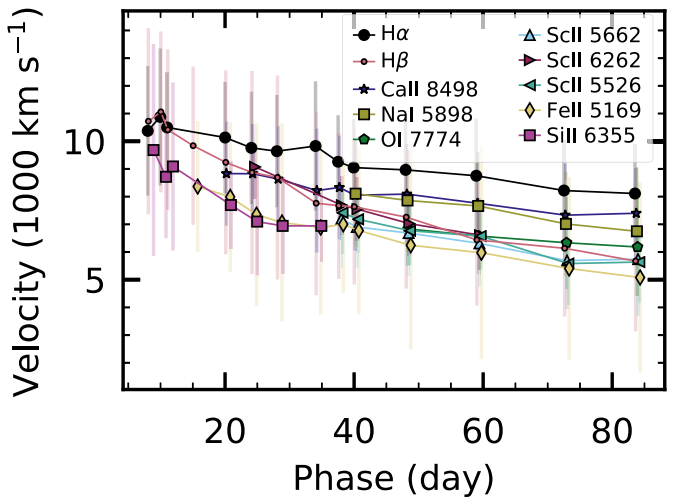

Figure 10. The evolution of the velocity of $\mathrm{H} \alpha, \mathrm{H} \beta$, Na I, O I, Ca II, Sc II, and Fe II over time. The points represent the minimum of the feature showing the velocity with the largest optical depth while the bars plot the range of velocities found in the line forming region. The coincidence of velocities of different species, especially at late times, indicates that mixing may have been important for ASASSN-15oz.

Filippenko (2012). For blended features we fit a multicomponent Gaussian to the feature. We use this method to fit $\mathrm{H} \alpha(\lambda 6561)$, O I $(\lambda 7774)$, Fe II ( $\lambda 5169)$, and Ca II $(\lambda \lambda 8498,8542,8662)$. Details of both fitting procedures are given in Appendix B. Rather than expressing an uncertainty on the fit, we calculate the range of velocities that encompass 68.2 per cent of the integrated (continuum subtracted) flux around the feature minimum. Because each line is formed in an extended region of the ejecta, this represents the range of velocities at which each line forms.

Fig. 10 shows the measured velocities. The distribution of velocities is typical of other IIP/IIL SNe representing the distribution of elements throughout the ejecta. A comparison of the velocities derived in this paper to the average values of 122 IIP/IIL SNe described in Gutiérrez et al. (2017) is shown in Fig. 11. The shaded regions represent the standard deviation of the sample. We find above average velocities throughout the ejecta.

\subsection{Infrared evolution}

Line identification was performed by comparing the NIR SOFI and SpeX spectra to those in Valenti et al. (2015) and Tomasella et al. (2018). Fig. 4 shows the NIR spectra with prominent features labelled. Like the optical, the first spectrum, taken $8 \mathrm{~d}$ post explosion (2018 September 05), is dominated by hydrogen features. The later spectra also mirror the optical evolution, developing metal lines as the ejecta slows and cools. We use the IR spectra to search for evidence of interaction via a high-velocity $\mathrm{He}$ I feature (see Section 6.2).

\subsection{Progenitor mass from nebular spectra}

Around $200 \mathrm{~d}$ the SN ejecta becomes optically transparent, revealing the inner core. At this time the SN enters the nebular phase, showing strong emission lines and no continuum (see Fig. 12). Analysis of the spectra at this time can constrain the geometry of the explosion as well as the abundance of different elements. In particular, spectral modelling of this stage is a powerful tool to constrain the nature of the progenitor. Jerkstrand et al. $(2012,2014)$ have shown that the intensities of a few lines are sensitive to the $M_{\text {ZAMS }}$ of the progenitor. Specifically, there is a tight monotonic correlation between the strength of the $[\mathrm{OI}](\lambda \lambda 6300,6334)$ line and the $M_{\text {ZAMS }}$ of the progenitor. This is because [OI] is more isolated and less sensitive to the explosive nucleosynthesis of the SN than the other lines and thus reflects the oxygen abundance of the progenitor which has been shown to correlate well with progenitor mass (Woosley \& Weaver 1995).

Jerkstrand et al. (2014) modelled nebular spectra for 12, 15, 19 , and $25 \mathrm{M}_{\odot}$ progenitors (M $M_{\text {ZAMS }}$ ). Starting with the explosion models from Woosley \& Heger (2007), the ejecta is divided into zones based on chemical composition and the evolution of the spectrum is found by modelling the gamma-ray transport and deposition, non-thermal electron degradation, NLTE ionization and excitation, and Monte Carlo radiative transfer.

Four nebular spectra of ASASSN-15oz were taken between day 228 (2016 April 11) and day 389 (2016 September 19). The first
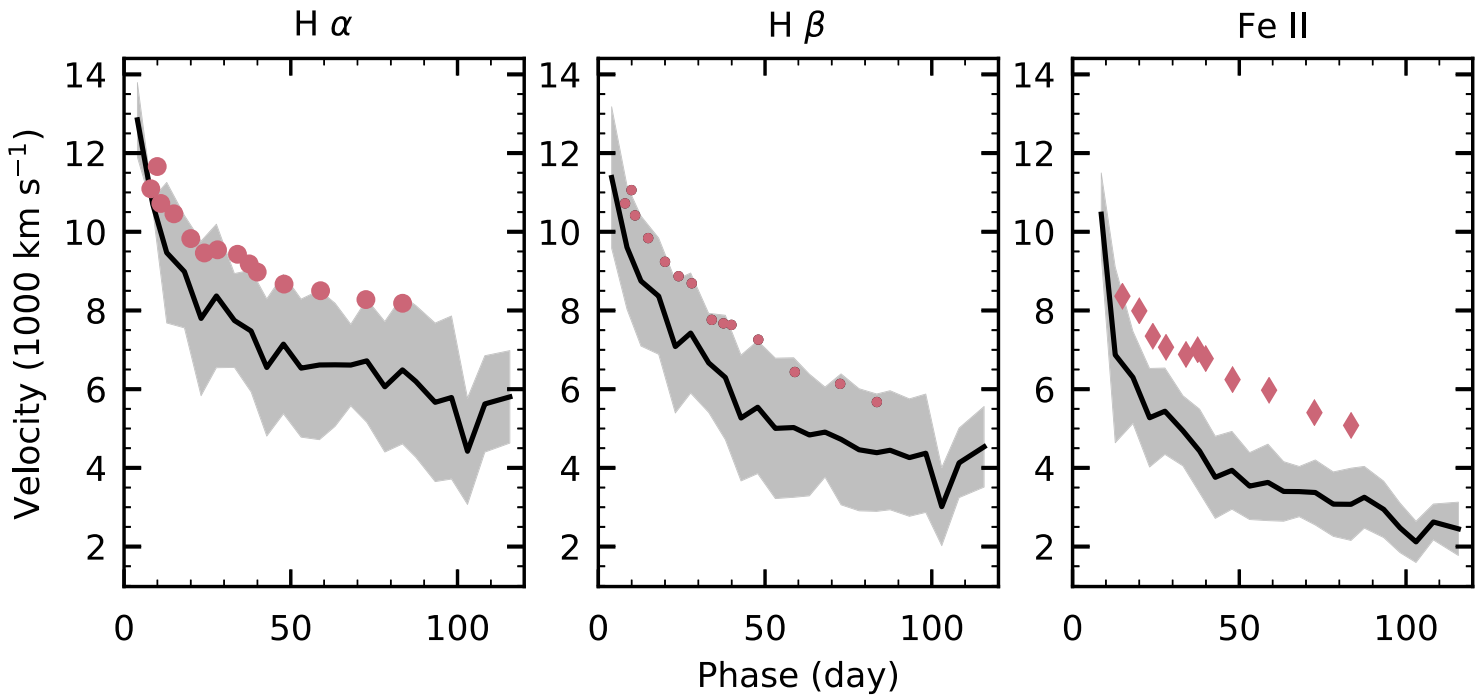

Figure 11. A comparison of the velocity of ASASSN-15oz (pink) to the mean velocity of 122 IIP/IIL SNe (Gutiérrez et al. 2017) (black) for H $\alpha$ (left), $\mathrm{H}$ $\beta$ (middle), and Fe II ( $\lambda 5169$; right). The grey regions represent the standard deviations of the mean velocities. The velocity of ASASSN-15oz is $>1 \sigma$ above average for all three ions. Each of these line originates in a different part of the ejecta indicating that this is a global trend and the explosion energy is above average. Following Silverman et al. (2012), we select velocity errors of $2 \AA$. These are contained within the symbols and so are not plotted. 


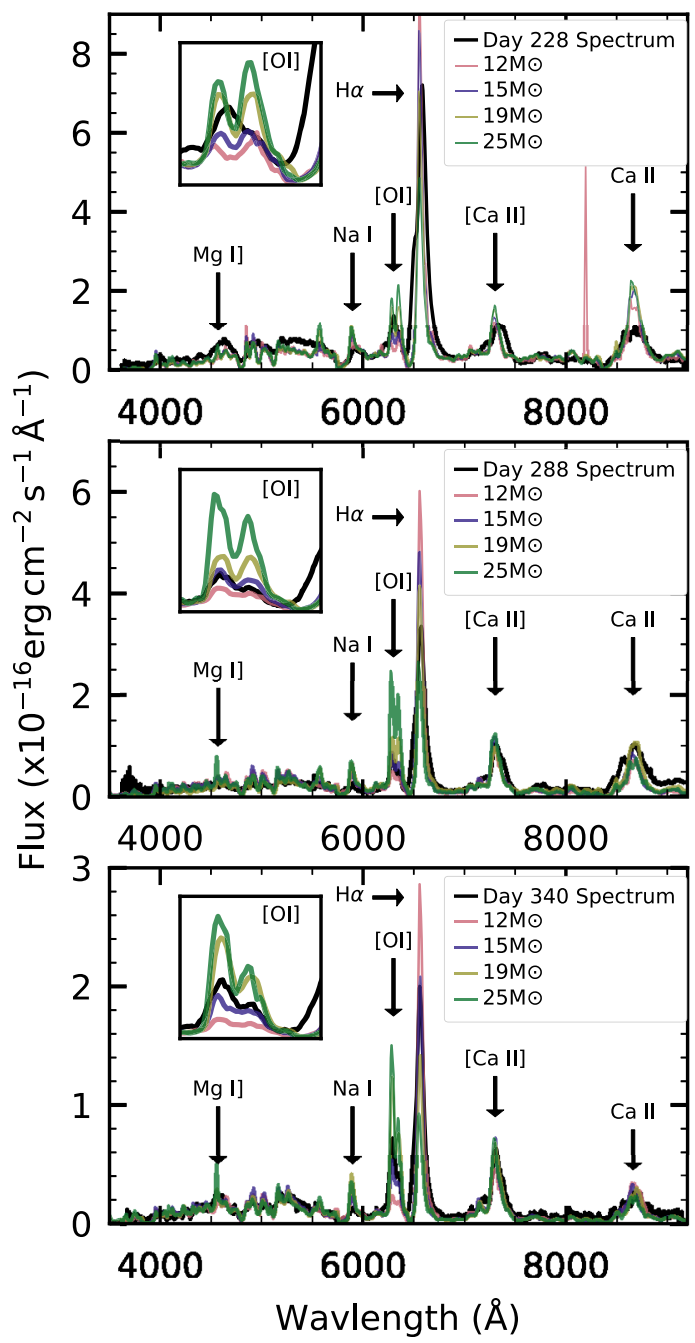

Figure 12. The nebular spectra of ASASSN-15oz from day 228 (2016 April 11; top), day 288 (2016 June 10; middle), and day 342 (2016 August 03; day 340; bottom) plotted with the scaled models of Jerkstrand et al. (2014) for $M_{\text {ZAMS }}=12,15,19$, and $25 \mathrm{M}_{\odot}$. Most features in the observed spectra are well matched by the models. The inset shows the [OI] $(\lambda \lambda 6300,6334)$ doublet, the strength of which increases monotonically with progenitor mass. The [O I] flux falls between the 15 and $19 \mathrm{M}_{\odot}$ models, consistent with the $17 \mathrm{M}_{\odot}$ progenitor mass found using light curve modelling in Section 6.4.

three spectra are high $\mathrm{S} / \mathrm{N}$ and are used for modelling. In Fig. 12 we compare these epochs to the different mass models, scaling the models to the observations over the full wavelength range. A zoom in on the [O I] line is displayed in the inset of each panel. We find the [OI] strength of ASASSN-15oz falls between the 15 and $19 \mathrm{M}_{\odot}$ models, consistent with the $17 \mathrm{M}_{\odot}$ found by modelling the light curve (see Section 6.4).

As a sanity check of the comparison between observations and synthetic spectra, we infer the ${ }^{56} \mathrm{Ni}$ mass from the scale factor we used to scale the models to the observations. Following Jerkstrand et al. (2018), the scaling combined with the equation for the luminosity of the cobalt decay (equation 6 of Jerkstrand et al. 2012) yields the ${ }^{56} \mathrm{Ni}$ mass (assuming complete trapping):

$\frac{F_{\mathrm{obs}}}{F_{\mathrm{mod}}}=\frac{d_{\mathrm{mod}}^{2}}{d_{\mathrm{obs}}^{2}} \frac{\left(M_{56, \mathrm{Ni}}\right)_{\mathrm{obs}}}{\left(M_{56, \mathrm{Ni}}\right)_{\mathrm{mod}}} \exp \left(\frac{t_{\mathrm{mod}}-t_{\mathrm{obs}}}{111.4}\right)$, where $F_{\text {obs }}$ and $F_{\text {mod }}$ are the observed and model fluxes, $d_{\text {obs }}$ and $d_{\text {mod }}$ the observed and model distances, $\left(M_{56, \mathrm{Ni}}\right)_{\text {obs }}$ and $\left(M_{56, \mathrm{Ni}}\right)_{\text {mod }}$ are the observed and model ${ }^{56} \mathrm{Ni}$ masses, and $t_{\mathrm{obs}}$ and $t_{\text {mod }}$ are phases of the observation and the model. Using this relation, we average the ${ }^{56} \mathrm{Ni}$ mass found for each mass model to find an ${ }^{56} \mathrm{Ni}$ mass of $0.03 \mathrm{M}_{\odot}$ for day 228 (2016 April 11) and day 288 (2016 June 10) and $0.02 \mathrm{M}_{\odot}$ for day 340 (2016 August 03). These values are a factor of four smaller than our lower limit for the ${ }^{56} \mathrm{Ni}$ mass (see Section 4.1). This is due at least in part to the fact that the spectral synthesis modelling does not account for the incomplete gammaray trapping in ASASSN-15oz. The fact that several IIP/IIL SNe do not have complete trapping (see Section 7) should be taken into account in the future.

\section{EVIDENCE FOR INTERACTION}

Although it is well known that massive stars undergo significant mass-loss during their lives, the details of this mass-loss (timing, rate, speed, geometry) are not well understood. The location and density of CSM around a star record the amount of mass lost at different times during a star's evolution. As the SN shock passes through this material, it provides a unique probe of this material and thus the mass-loss of the progenitor. While the mass-loss of stars with dense CSM (e.g. IIn SNe) that show strong evidence of interaction, such as narrow emission lines in photospheric spectra or irregular photometric brightening, is often studied, the mass-loss of SNe with lower density CSM is often ignored.

In IIP-like $\mathrm{SNe}$, the plateau is the result of the recession of the photosphere due to hydrogen recombination matching the expansion of the SN ejecta. The linear decline of the light curves of IIL-like $\mathrm{SNe}$ is then explained by the photosphere receding faster than the ejecta's expansion. The photosphere's recession is governed by the hydrogen density profile. Although there are a number of ways to alter the density profile (e.g. mixing of the $\mathrm{H}$ and $\mathrm{He}$ envelopes; Utrobin \& Chugai 2017), one possibility is that mass-loss during the last stage of stellar evolution produces a shallow density profile. In this scenario IIL-like SNe should undergo more mass-loss than IIP-like SNe. ASASSN-15oz, a IIL-like SN with photometric and spectroscopic observations from the X-ray through the radio is the ideal SN on which to study the importance of mass-loss for IIPlike and IIL-like SNe. The different epochs and wavelengths probe different periods of mass-loss allowing for the characterization of the mass-loss history of the progenitor. In this section we analyse the evidence for interaction at different wavelengths. We note that we cannot search for flash ionization features as there are no spectroscopic observations until near maximum.

\subsection{UV and X-ray}

ASASSN-15oz is not detected in any of the Swift X-ray observations. The flux limits were converted to luminosity limits assuming Galactic absorption of $6.18 \times 10^{20} \mathrm{~cm}^{-2}$ (Kalberla et al. 2005) and a distance of $28.83 \mathrm{Mpc}$.

We compare these upper limits to the model of SN 1999em (Chugai, Chevalier \& Utrobin 2007) which has similar progenitor and explosion parameters. They model the interaction as an infinitely thin double-shock structure (Chevalier 1982; Nadyozhin 1985) composed of a forward shock propagating into the surrounding gas and reverse shock propagating backwards into the SN ejecta, towards its core. Chugai et al. (2007) find that the reverse shock dominates the $\mathrm{X}$-ray observations for a pre-explosion mass-loss rate of $10^{-6} \mathrm{M}_{\odot} \mathrm{yr}^{-1}$ and a wind velocity of $10 \mathrm{~km} \mathrm{~s}^{-1}$ 
producing luminosities between $10^{38}$ and $10^{39} \mathrm{erg} \mathrm{s}^{-1}$. The forward shock produces luminosities comparable to the reverse shock in the first $10 \mathrm{~d}$ and decreasing to below $10^{34} \mathrm{erg} \mathrm{s}^{-1}$ by day 100 . Our non-detections are above the upper limit of this range, making it plausible that if there is X-ray emission, it is below our detection limits.

We use $\mathrm{SYN}++$ to model and tentatively identify absorption features from Fe II, Ti II, and Mg II features in the first Swift UV grism spectrum (see Fig. 3). This spectrum shows no sign of the narrow emission lines typically associated with strong interaction $\left(\dot{M}>10^{-4} \mathrm{~g} \mathrm{~cm}^{-1}\right.$; Kiewe et al. 2012)

\subsection{Optical spectroscopy}

Chugai et al. (2007) proposed a high-velocity hydrogen and helium line are produced by interaction of the SN ejecta with the stellar wind. According to their model, a notch on the blue side of the $\mathrm{H}$ $\alpha$ profile should appear 40 to $80 \mathrm{~d}$ post explosion. Although they could construct a model with the introduction of a cold dense shell in which a similar notch was visible in $\mathrm{H} \beta$ their simplest model did not show $\mathrm{H} \beta$ due to its low opacity in the wind. They do however, predict absorption in He I $\lambda 1083020-60 \mathrm{~d}$ post explosion, a line that is only excited when wind is present.

A feature blueward of $\mathrm{H} \alpha$ is often present in Type II SNe, however, it is unclear if this feature is due to high-velocity hydrogen or to Si II ( 26355$)$ and it has thus been named the cachito feature to avoid association with a particular species (Gutiérrez et al. 2017). Building on the analysis of smaller samples (e.g. Leonard et al. 2002; Inserra et al. 2013; Valenti et al. 2014), Gutiérrez et al. (2017) searched for this feature in 122 IIP/IIL SNe. They find the cachito feature in $70 \mathrm{SNe}$ in their sample and divide the detections in early phase detections (phase $<40 \mathrm{~d}$ ) and late phase detections (phase $>40 \mathrm{~d}$ ). The velocities of the cachito feature observed in the early sample are most often well matched to the Fe II velocity if interpreted as Si II. For this reason, they interpret the cachito feature in the early sample as Si II and at late times as high-velocity hydrogen. Fig. 13 shows the time evolution of the region surrounding $\mathrm{H} \alpha$ (left-hand panel), $\mathrm{H} \beta$ (middle panel), and $\mathrm{He}$ I (right-hand panel). $\mathrm{H} \alpha$ and $\mathrm{H} \beta$ are marked with a brick dotted line in the left and middle panels, respectively. The dashed line in the left-hand panel marks the location of the cachito feature and in the middle panel, the expected location of the cachito feature in $\mathrm{H} \beta$. The time of expected CSM interaction is marked in grey. ASASSN-15oz shows a strong cachito feature in the first spectrum, $8 \mathrm{~d}$ post explosion. This feature strengthens until about day 15 and then fades until it is no longer visible at day 60 . We find no evidence for a highvelocity feature in $\mathrm{H} \beta$. The $\mathrm{He}$ I feature is fully blended with CI ( 2 10691) and Pa- $\gamma$ and if it is present, it is impossible to deblend and identify. A comparison of the cachito velocity, when interpreted as Si II, and the Fe II velocity is plotted in Fig. 14. We find excellent agreement between the two velocities strengthening our conclusion that this feature is due to Si II rather than high-velocity hydrogen.

The $\mathrm{H} \alpha$ emission profiles in the nebular spectra of ASASSN$15 \mathrm{oz}$ are asymmetric (see Fig. 15). In addition to emission at the rest wavelength of $\mathrm{H} \alpha$, there is evidence of both blueshifted and redshifted emission at 2200 and $800 \mathrm{~km} \mathrm{~s}^{-1}$, respectively. This feature is strongest in the first spectrum at day 228 (2016 April 11), becoming weaker in the subsequent spectra. Asymmetric $\mathrm{H} \alpha$ emission has been seen in a few IIP/IIL SNe (e.g. 1999em: Leonard et al. 2002; SN 2004dj: Chugai et al. 2005; SN 2013ej: Utrobin \&

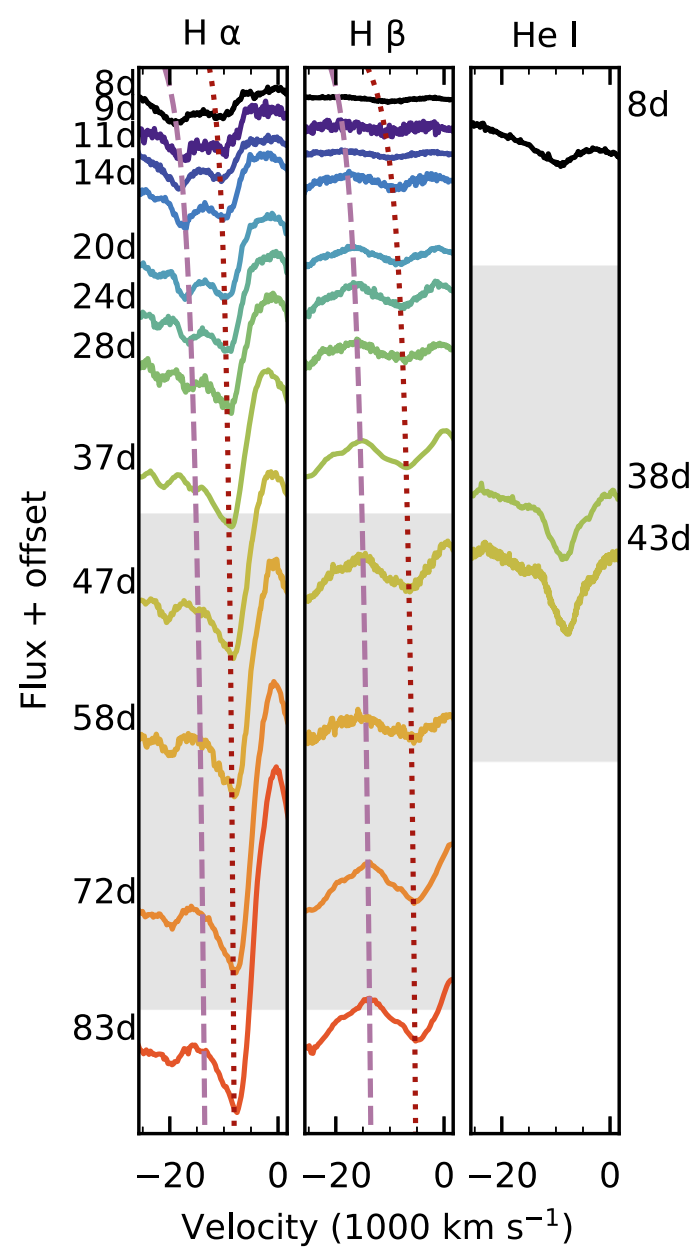

Figure 13. The evolution of the cachito feature (left-hand panel; dashed line) over time. The feature is visible bluewards of $\mathrm{H} \alpha$ (left-hand panel; dotted line) in the first spectrum, $8 \mathrm{~d}$ post explosion. It increases in strength for the next $10 \mathrm{~d}$, then decreases until it is barely visible at $60 \mathrm{~d}$ post explosion. This evolution is counter to the evolution described in Chugai et al. (2007) in which the high-velocity hydrogen feature becomes visible around day 40, making it unlikely that it is high-velocity hydrogen. There is no evidence of a high-velocity hydrogen feature in $\mathrm{H} \beta$ (centre panel; $\mathrm{H} \beta$ marked with dotted line; cachito velocity marked with dashed line) although this is not surprising given the low opacities predicted by Chugai et al. (2007). The high-velocity He I ( $\lambda 10830)$ feature (right-hand panel) also predicted by the models of Chugai et al. (2007) is too heavily blended with the $\mathrm{Pa}-\gamma$ and $\mathrm{C}$ I lines to be identifiable.

Chugai 2017). Leonard et al. (2002) explain the shape of the $\mathrm{H}$ $\alpha$ emission as either due to an asymmetric line-emitting region or due to interaction between the ejecta and the CSM while Chugai et al. (2005) and Utrobin \& Chugai (2017) attribute this feature to a bipolar ${ }^{56} \mathrm{Ni}$ ejecta.

\subsection{Radio}

The origin of radio emission from $\mathrm{SNe}$ can be explained by the interaction of the SN ejecta with the CSM. This interaction leads to a shockwave traveling via the CSM that in turn accelerates electrons and enhances magnetic fields and thus synchrotron emission ensues (Chevalier 1982, 1998; Weiler et al. 2002; Chevalier \& Fransson 2006). It has been shown over the last decade that in most observed 


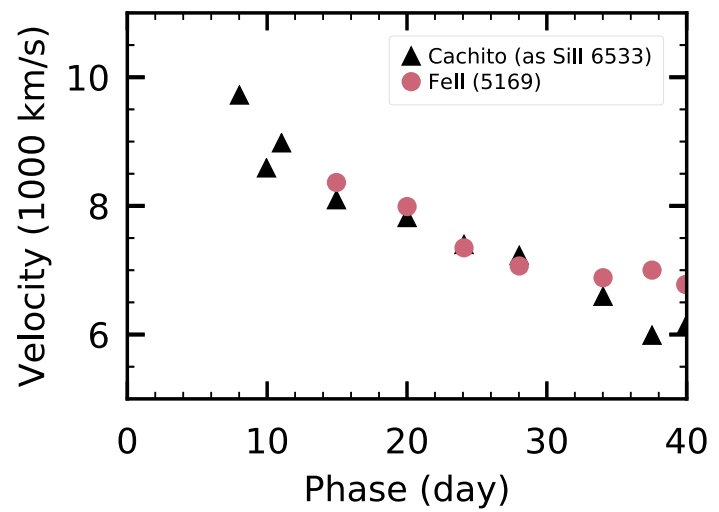

Figure 14. A comparison of the velocity of the cachito feature if it is $\mathrm{Si}$ II ( $\lambda 6355)$ (black triangles) and the velocity of the metals in the ejecta as characterized by the Fe II ( $\lambda 5169$ lines; pink circles). The velocities are the same indicating that this line is likely due to $\mathrm{Si}$ II rather than high-velocity hydrogen in the circumstellar medium.

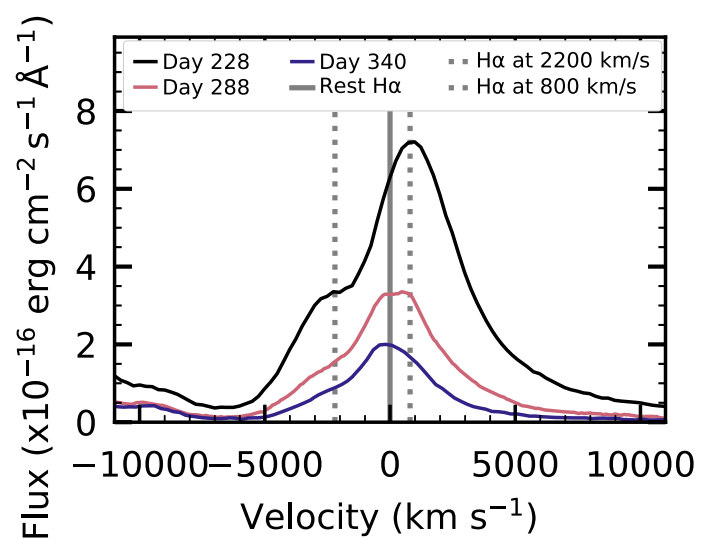

Figure 15. The asymmetric $\mathrm{H} \alpha$ emission in the nebular spectra of ASASSN-15oz. The spectrum shows $\mathrm{H} \alpha$ emission at rest (solid grey line), blueshifted by $2200 \mathrm{~km} \mathrm{~s}^{-1}$ and redshifted by $800 \mathrm{~km} \mathrm{~s}^{-1}$ (dashed grey lines). Although the asymmetry is visible in all of the spectra, it is most prominent in the earliest nebular spectrum on day 228 (2016 April 11) and could be a sign of interaction with CSM. However such strong asymmetry would require an high level of interaction not visible by other probes.

$\mathrm{SNe}$, the optically thick part of the observed radio spectrum can be modelled by synchrotron self-absorption (SSA; e.g. Soderberg et al. 2012; Horesh et al. 2013). We use the Chevalier (1998) SSA formalism to model the radio measurements of ASASSN-15oz. In such a model the radio emission peaks at a frequency below which the emission is strongly absorbed by SSA. Above that frequency the emission becomes optically thin. As the shockwave progress outwards to lower CSM densities, the SSA optical depth drops and the peak of the radio emission moves to lower and lower frequencies. We use Equation 1 from Chevalier (1998) to model the radio dataset (see Fig. 16). We use the standard equipartition assumption and set the microphysical parameters, $\epsilon_{\mathrm{e}}=\epsilon_{\mathrm{B}}=0.1$ (Chevalier \& Fransson 2006). We also set the electron power-law distribution to $\mathrm{p}=3$, leaving the CSM density and shockwave velocity as free parameters. We find the shockwave velocity (assuming constant velocity) is $\sim 1.4 \times 10^{4} \mathrm{~km} \mathrm{~s}^{-1}$ (which is the typical average value observed in SNe; e.g. Chevalier \& Fransson 2006). Assuming the

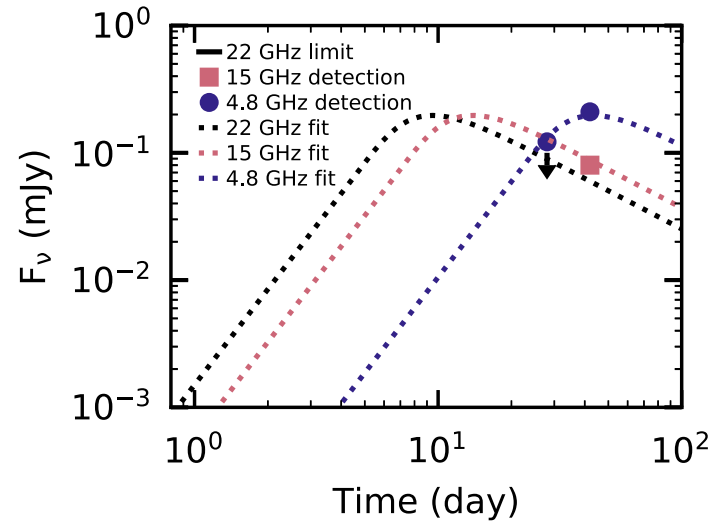

Figure 16. The best-fitting SSA model (dotted lines) plotted over the observations (symbols) at $22 \mathrm{GHz}$ (black), $15 \mathrm{GHz}$ (pink), and $4.8 \mathrm{GHz}$ (indigo). These data are best fit by a shock velocity of $\sim 1.4 \times 10^{4} \mathrm{~km} \mathrm{~s}^{-1}$ and a density of $\mathrm{K}=4.51 \times 10^{11} \mathrm{~g} \mathrm{~cm}^{-1}$.

CSM surrounding the $\mathrm{SN}$ is created by a stellar wind from the progenitor prior to explosion, we model the density profile of the $\mathrm{CSM}$ as

$\rho(r)=\frac{\dot{M}}{4 \pi r^{2} v_{\text {wind }}}=\frac{K}{r^{2}}$,

where $\dot{M}$ is the wind mass-loss rate and $v_{\text {wind }}$ is the wind velocity. We find $\mathrm{K}=4.51 \times 10^{11} \mathrm{~g} \mathrm{~cm}^{-1}$. With a wind velocity between 10 and $100 \mathrm{~km} \mathrm{~s}^{-1}$, this implies a mass-loss rate between $\dot{M} \sim 0.9$ and $9 \times 10^{-7} \mathrm{M}_{\odot} \mathrm{yr}^{-1}$.

\subsection{Light-curve modelling with SNEC}

We fit the light curve of ASASSN-15oz using the Supernova Explosion Code (SNEC) (Morozova et al. 2015). SNEC is an open source Lagrangian 1D radiation hydrodynamic code that employs flux-limited radiation diffusion and assumes local thermodynamic equilibrium (LTE). SNEC uses a Paczyński equation of state (Paczynski 1983) solving for the ionization fractions using the Saha equations in the non-degenerate approximation (Zaghloul, Bourham \& Doster 2000). Opacities are drawn from OPAL type II opacity tables (Iglesias \& Rogers 1996) at high temperatures ( $\mathrm{T}=10^{4.5}$ to $10^{8.7} \mathrm{~K}$ ) and tables of Ferguson et al. (2005) at low temperatures $\left(\mathrm{T}=10^{2.7}\right.$ to $10^{4.5} \mathrm{~K}$ ) supplemented with an opacity floor of $0.01 \mathrm{~cm}^{2} \mathrm{~g}^{-1}$. The mixing of isotopes due to the explosion is modelled by boxcar smoothing the progenitor composition profile with a kernel of $0.4 \mathrm{M}_{\odot}$.

In the past, hydrodynamic SN models that used progenitors evolved with stellar evolution codes (referred to as evolutionary progenitors for the remainder of this paper; e.g. KEPLER code, Weaver, Zimmerman \& Woosley 1978; Woosley \& Heger 2007, 2015; Sukhbold \& Woosley 2014; Sukhbold et al. 2016; MESA, Paxton et al. 2018) failed to reproduce the shape of the early observed light curves of Type II SNe. Some authors have solved this issue by using hydrostatic progenitor models with extended envelopes and density profiles that are set to match observations rather than calculated by stellar evolution codes (referred to as non-evolutionary progenitors for the remainder of this paper; e.g. Utrobin \& Chugai 2008, Utrobin \& Chugai 2017). However, adding a dense CSM ejected just before the explosion to progenitors evolved with stellar evolution codes has resolved the discrepancy 
Table 2. The grid of parameters used by SNEC. The values that best fit the data are in bold. The pre-explosion masses are taken from table 2 of Sukhbold et al. (2016).

\begin{tabular}{ll}
\hline Parameter & Values \\
\hline Progenitor ZAMS mass $\left(M_{\text {ZAMS }} ; \mathrm{M}_{\odot}\right)$ & $11,13,14,16, \mathbf{1 7}, 18,21$ \\
Final pre-SN mass $\left(\mathrm{M}_{\odot}\right)$ & $10.688,11.567,12.079,13.145, \mathbf{1 4 . 3 0 1}, 14.936,16.119$ \\
Pre-SN radius $\left(100 \mathrm{R}_{\odot}\right)$ & $5.7,7.0,7.8,8.9, \mathbf{9 . 1}, 9.7,11.2$ \\
Explosion energy $\left(E_{\text {exp }} ; 10^{51} \mathrm{erg}\right)$ & $0.5,0.8,1.1, \mathbf{1 . 4}, 1.7,2.0$ \\
CSM density $\left(\mathrm{K} ; 10^{17} \mathrm{~g} \mathrm{~cm}^{-1}\right)$ & $0,10,20,30,35, \mathbf{4 0}, 50,60$ \\
CSM extent $\left(R_{\text {ext }} ; 100 \mathrm{R}_{\odot}\right)$ & $0,15, \mathbf{1 8}, 21,24,27,30,33$ \\
Ni mass $\left(\mathrm{M}_{\odot}\right)$ & $\mathbf{0 . 0 8}, 0.09,0.11$ \\
\hline
\end{tabular}

between the early observed light curves and the light curves modelled using physically derived progenitors (e.g. Gezari et al. 2015; Förster et al. 2018; Morozova et al. 2018). The presence of this CSM is corroborated by other observations of IIP/IIL SNe (e.g. Yaron et al. 2017; Bullivant et al. 2018).

For the progenitor models, we use a set of non-rotating solar metallicity RSGs parametrized by the $M_{\text {ZAMS }}$, evolved with the KEPLER code and described in Sukhbold et al. (2016). Note that the stellar evolution calculations made with KEPLER take into account the regular steady winds observed in RSGs, in the way prescribed by Nieuwenhuijzen \& de Jager (1990) and Wellstein \& Langer (1999). For this reason, the final pre-SN masses of the models may be up to several $\mathrm{M}_{\odot}$ smaller than their initial $M_{\text {ZAMS }}$. Both the $M_{\text {ZAMS }}$ and pre-SN masses of the models used are given in Table 2. However, for the typical mass-loss rates of $\lesssim 10^{-6} \mathrm{M}_{\odot} \mathrm{yr}^{-1}$, the density in these regular steady winds is too low to have any noticeable effect on the post-explosion optical light curves, and these winds are not included in the progenitor profiles.

We explore variations in CSM due to enhanced mass-loss by adding a steady-state wind above the RSG models with the density profile expressed in equation (3). This approximation allows us to parametrize the CSM with two parameters, $K$ and $R_{\text {ext }}$, the radial extent of the CSM. In reality, the late enhanced mass-loss does not have to be in the form of a steady wind with constant $\dot{M}$ and may instead represent one or several eruptive outbursts. However, in our experience, the early optical light curve depends weakly on the exact slope of the CSM profile, being more sensitive to the total CSM mass.

Recently, Paxton et al. (2018) demonstrated that IIP/IIL SNe are well characterized by the radiation diffusion approximation from shock breakout through the fall from plateau. At the same time, they have shown that the assumption of LTE is not well satisfied at the photosphere location of the IIP/IIL SNe models. For this reason, SNEC colour light curves generally rise faster than the light curves obtained from more sophisticated multigroup radiationhydrodynamics codes, like STELLA (Paxton et al. 2018). However, this fact makes the case for introducing a CSM surrounding the RSG before its explosion even stronger. In this view, the total CSM mass derived in our analysis may be considered as a lower limit, while the values obtained with more advanced codes should be comparable, or in some cases larger (Moriya et al. 2017; Paxton et al. 2018).

To account for the formation of the neutron star, we excise the inner $1.4 \mathrm{M}_{\odot}$ of the progenitor models prior to the explosion. After that, we model the explosion of ASASSN-15oz using a thermal bomb with explosion energy $E_{\text {exp }}$ lasting for one second in the inner $0.02 \mathrm{M}_{\odot}$. When computing the amount of energy injected in the form of a thermal bomb, SNEC automatically takes into account the total initial (negative, mostly gravitational) energy of the models. Therefore, by the explosion energy $E_{\exp }$ in our analysis we mean the total energy of the models after explosion, which is conserved in the code to better than 1 per cent accuracy and eventually mainly transformed into the kinetic energy of the expanding envelope.

SNEC does not model nuclear reaction networks, but rather takes as input a mass of ${ }^{56} \mathrm{Ni}$. We use a grid of ${ }^{56} \mathrm{Ni}$ masses informed by our analysis in Section 3. While SNEC allows for the ${ }^{56} \mathrm{Ni}$ to be mixed out to different values of mass coordinate, Morozova, Piro \& Valenti (2017) find that the progenitor masses and explosion energies derived from fitting the IIP/IIL SN light curves are not very sensitive to the degree of ${ }^{56} \mathrm{Ni}$ mixing. Therefore, in our study we choose to keep this parameter fixed and mix the ${ }^{56} \mathrm{Ni}$ up to $5.0 \mathrm{M}_{\odot}$.

We use SNEC to find the best progenitor parameters varying the progenitor mass, explosion energy, ${ }^{56} \mathrm{Ni}$ mass, CSM density, and CSM extent. Table 2 gives the set of parameters used, resulting in over 5000 model light curves. Morozova et al. (2018) find the best fit model with and without CSM as a two-step process. First they modelling the second half of the light curve, characterized by the $s_{2}$ slope without CSM to determine the best progenitor mass and explosion energy. Then, fixing the explosion energy and progenitor mass, they explore the CSM parameter space. This is computationally less intensive than modelling the full parameter space and allows them to explore a finer grid of parameters. Given the complexity of the parameter space, we choose to model the best light curve with CSM, exploring the full set of parameters simultaneously.

For ease of comparison with observations, SNEC uses the photospheric temperature at each time-step to compute a blackbody spectrum, which it combines with different filter throughputs to output a light curve in Sloan filters $u, g, r$, and $i$, Bessel filters $U$, $B, V, R$, and $I$ and PanSTARRS filter $z$. During the rise and plateau phase, a blackbody should be a good approximation to the longer wavelengths. However, line blanketing may cause the bluer filters to be a poor representation of the observed spectrum (Dessart \& Hillier 2005; Kasen \& Woosley 2009). For this reason the bestfitting model is determined using the $g, r$, and $i$ filters. While we do have a $V$-band light curve, the throughput heavily overlaps with the $g$ and $r$ bands and its inclusion would give more weight to these wavelengths without providing new information. The best fit model is determined by interpolating the well-sampled model to the observed wavelengths and computing a chi-square minimization across all three filters. Given the uncertainty in the explosion time, we shift the model spectrum by $\pm 4 \mathrm{~d}$ and treat this offset as a free 
Table 3. The best-fitting SNEC model parameters for the simultaneous fits to $g, r$, and $i$ bands and to the bolometric luminosity with and without CSM.

\begin{tabular}{lcccc}
\hline \multirow{2}{*}{ Parameter } & \multicolumn{2}{c}{ Fit to $g, r$ i bands } & \multicolumn{2}{c}{ Fit to bolometric luminosity } \\
& With CSM & Without CSM & With CSM & Without CSM \\
\hline Progenitor ZAMS mass $\left(M_{\text {ZAMS }} ; \mathrm{M}_{\odot}\right)$ & 17 & 18 & 18 & 17 \\
Final pre-SN mass $\left(\mathrm{M}_{\odot}\right)$ & 14.301 & 14.936 & 14.936 & 14.301 \\
Pre-SN radius $\left(100 \mathrm{R}_{\odot}\right)$ & 9.1 & 9.7 & 9.7 & 9.1 \\
Explosion energy $\left(E_{\text {exp }} ; 10^{51}\right.$ ergs $)$ & 1.4 & 2.0 & 1.4 & 2.0 \\
CSM density $\left(\mathrm{K} ; 10^{17} \mathrm{~g} \mathrm{~cm}^{-1}\right)$ & 40 & 0 & 60 & 0 \\
$\mathrm{CSM}$ extent $\left(R_{\text {ext }} ; 100 \mathrm{R}_{\odot}\right)$ & 18 & 0 & 15 & 0 \\
Ni mass $\left(\mathrm{M}_{\odot}\right)$ & 0.083 & 0.083 & 0.083 & 0.083 \\
Total CSM mass $\left(\mathrm{M}_{\odot}\right)$ & 1.5 & 0 & 1.4 & 0 \\
Time offset $\left(t_{\text {offset }} ; \mathrm{d}\right)$ & 3 & 3 & 2 & 1 \\
\hline
\end{tabular}

parameter $\left(t_{\text {offset }}\right)$. Parameters for the best-fitting models with and without CSM are given in Table 3. These models are shown with (solid lines) and without (dashed lines) CSM interaction in the top panel of Fig. 17. Integrating the CSM density over it radial extent, we find a total CSM mass of $1.5 \mathrm{M}_{\odot}$.

As a sanity check, we derive the bolometric luminosity by fitting a blackbody to the photometric observations and find the best fit SNEC model with and without CSM. The parameters for these models are given in Table 3 and are plotted over the bolometric luminosity in the bottom panel of Fig. 17. The explosion energy and ejecta masses are similar to those derived from a simultaneous fit of the $g, r$, and $i$ bands. Although the individual CSM parameters vary, these are highly degenerate and the total CSM mass, which our model is a better measure of, is very similar. The difference in the total CSM mass from the two different methods can be seen as an indication of the uncertainty in this value.

Towards a consistent picture of the SN explosion, we also verified that the parameters we found from the light curve modelling were in agreement with the interpretation of other observables. The high velocity derived from the spectroscopy is consistent with the large mass and explosion energy found by the light curve fitting. It is also compatible with the incomplete gamma-ray trapping, which is expected in $\mathrm{SNe}$ with high core velocities (Jerkstrand, Fransson \& Kozma 2011). Fig. 18 shows a comparison of the photospheric velocity of the best-fitting SNEC model to the observed photospheric velocity of ASASSN-15oz. Following Faran et al. (2014), we define the photospheric velocity as that of Fe II ( $\lambda 5169)$. We use cachito feature, now identified as Si II ( 26355$)$, to trace the photosphere at early times. We note that while the Fe II ( $\lambda 5169)$ is often used to characterize the photospheric velocity, it is not well understood where in the atmosphere line is being formed. Paxton et al. (2018) find that the Fe II ( $\lambda 5169)$ line is originating above the photosphere at a specific value of Sobolev optical depth ( $\left.\tau_{\text {sob }}\right)$ (they find $\tau_{\text {sob }} \sim 1$ fits the observations well). However, Hamuy (2001), in comparing the velocity derived from the Fe II ( 25169$)$ line to the photospheric velocity in the Type II SN atmospheric models of Eastman, Schmidt \& Kirshner (1996), find a systematic offset for individual objects that is in different directions for different objects, leading to no average offset. While there is fair agreement between the our model and observe velocity at early times, the model velocity is significantly lower than the observed velocity starting around day 25. Following Paxton et al. (2018), we adjust our model velocity to the value at $\tau_{\text {sob }}=1.0$. Fig. 18 shows that this adjustment brings the SNEC velocities closer to the observed velocities. A more detailed discussion concerning the modelling

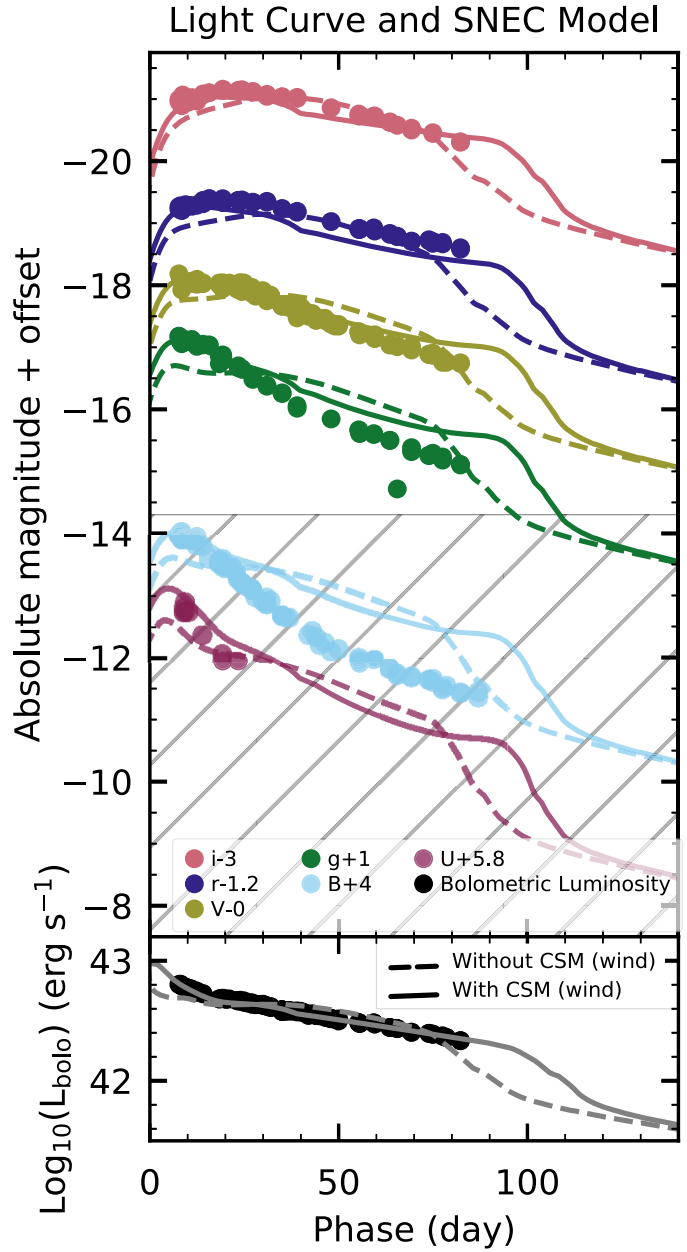

Figure 17. Top: The multiband optical light curve of ASASSN-15oz. The observations are shown as circles and the best-fitting SNEC model is shown with and without CSM (solid and dashed lines, respectively). We require CSM to fit the light curve at early times. The blue filters are affected by line blanketing ( $U$ and $V$ bands) and are thus not expected to be well approximated by the blackbody approximation used to convert the bolometric luminosity produced by the modelling into magnitudes in individual filters. For this reason we do not use these in the fit and have placed hatched lines over them. Line blanketing may also be responsible for the overestimate of the model flux in the $g$ band. Bottom: the bolometric luminosity of ASASSN-15oz plotted with the best model fit to the bolometric luminosity including CSM (solid line) and the best model without CSM (dashed line). 


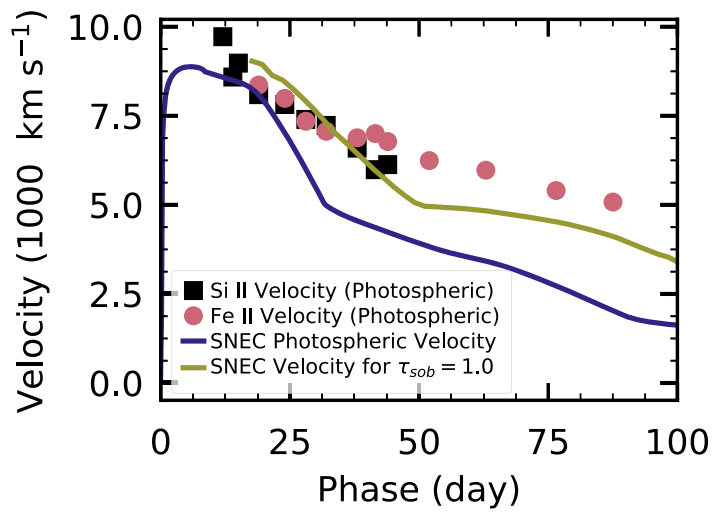

Figure 18. The model photospheric velocity computed by SNEC (indigo line) compared to the observed photospheric velocity (symbols) taken to be the velocity of the Si II ( $\lambda 6355)$ feature at early times (black squares) and Fe II ( $\lambda 5169)$ feature at later times (pink circles). The model deviates from the observations around the time that the photosphere begins to recede into the ejecta. The model velocity at $\tau_{\text {sob }}=1$ is plotted in yellow. While this provides a better agreement with the observations, the model velocity evolution is still faster than the observed evolution.

of the minimum of the Fe II line is beyond the scope of this paper.

According to the classical model by Chevalier (1982), the interaction of the $\mathrm{SN}$ ejecta with the surrounding wind leads to the formation of a cool dense shell between the forward and the reverse propagating shock waves. This shell is responsible for the X-ray emission from Type II SNe. In addition, an opaque shell can be formed in front of the forward shock wave, which may serve as an explanation of the narrow emission lines with broad wings seen in some interacting SNe (Chugai 2001). In our SNEC model, the CSM density is too high and the density drop between the envelope and the CSM is not sufficient to form a significantly overdense shell between the forward and reverse shock waves. This is supported by Fig. 19, where the interface between the RSG envelope and the CSM corresponds to the velocity coordinate of $\sim 4200 \mathrm{~km} \mathrm{~s}^{-1}$. In this model, the forward shock wave sweeps the CSM almost entirely before the breakout, and the opaque circumstellar shell ahead of the shock wave is not formed either.

On the other hand, if we were to consider a three-component model, consisting of an RSG, a dense CSM, and a regular lowdensity stellar wind, like in Morozova et al. (2018), the thin cool dense shell would be formed at the interface between the dense CSM and the wind. In this scenario, the forward shock wave keeps propagating into the low density wind and generates radio emission. Since SNEC is not yet capable of handling the low density stellar wind, we have omitted it in our current simulations. However, we already know from the radio observations (Section 6.3) that the massloss rate of this wind in ASASSN-15oz is $\sim 0.9-9 \times 10^{-7} \mathrm{M}_{\odot} \mathrm{yr}^{-1}$. Such low-density wind would not have any significant influence on the optical light curve of the SN, which justifies using a twocomponent model (RSG and dense CSM only) for the purpose of this section.

\section{DISCUSSION}

It is useful to compare observations of ASASSN-15oz to those of other IIP/IIL SNe to understand if it is a typical IIL-like SN or a

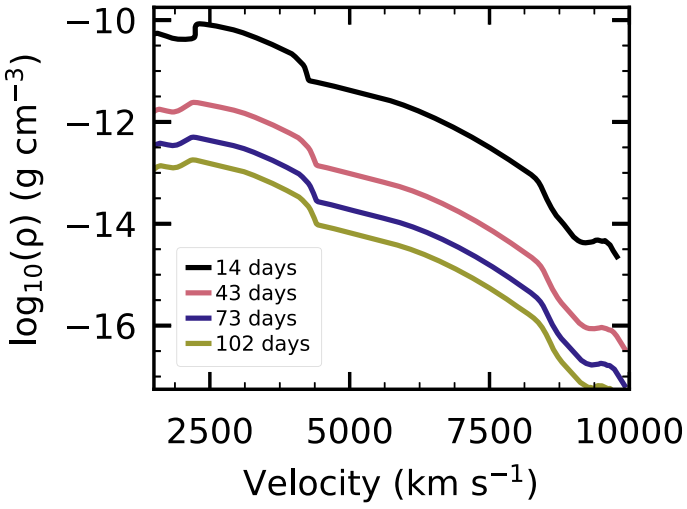

Figure 19. Density as a function of velocity in our best-fitting SNEC model at the free expansion stage. The interface between the RSG envelope and the CSM corresponds to the velocity value of $\sim 4200 \mathrm{~km} \mathrm{~s}^{-1}$. The kink in the velocity evolution at $5000 \mathrm{~km} \mathrm{~s}^{-1}$ is related to the fact that we do not attempt to artificially smooth the density step at the interface between the RSG model and the attached CSM. In reality, this interface will likely be smooth, but its exact shape will depend on the formation mechanism of the CSM, which is currently unknown. The mild increase in density at $\sim 2300 \mathrm{~km} \mathrm{~s}^{-1}$ is a result of the reverse shock wave reflected from this interface.

unique event. Although they are part of a continuous class, IILlike SNe typically have higher velocity ejecta, are brighter, and have steeper light curve slopes than IIP-like SN. In Section 5.1, we showed that ASASSN-15oz has above average ejecta velocity. Here we examine the light-curve slope and absolute magnitude in the context of a sample of IIP/IIL SNe.

Population studies of IIP/IIL SNe have found that $\mathrm{SNe}$ with steeper light curves are brighter (Li et al. 2011; Anderson et al. 2014; Sanders et al. 2015; Valenti et al. 2016), due to a larger explosion energy, a larger progenitor, CSM interaction, or a combined effect. The left-hand panel of Fig. 20 shows the $V$-band light curve of ASASSN-15oz compared to nine other well-studied SNe with a range of $s_{50 \mathrm{~V}}$. ASASSN-15oz is brighter and more steeply declining than the classic IIP-like SNe 1999em and 2014et. We also note that the slope of the radioactive tail of ASASSN-15oz is steeper than other objects plotted here.

We use our database of publicly available light curves, SNDAVIS, to compare ASASSN-15oz to a larger sample of objects. In the right-hand panel of Fig. 20 we plot the $s_{50 \mathrm{~V}}$ and the absolute $V$-band magnitude for $105 \mathrm{SNe}$. As a bright IIL-like SN, ASASSN-15oz is consistent with the sample correlation.

We also compare $s_{50 \mathrm{~V}}$ and the radioactive decay slope for ASASSN-15oz with all SNe in our database for which both slopes are measured in Fig. 21. The steepness of $s_{50 \mathrm{~V}}$ in a IIP/IIL SN light curve can be explained by the hydrogen recombination front receding faster than the ejecta expansion, implying a progenitor with a smaller hydrogen envelope. The slope of the radioactive decay tail is determined by the fraction of gamma rays trapped and reprocessed by this same hydrogen envelope. A lower fraction of trapped gamma rays can result either from a smaller hydrogen envelope or by external mixing of ${ }^{56} \mathrm{Ni}$. Given the possible dependence of both $s_{50 \mathrm{~V}}$ and the radioactive decay slope on the hydrogen envelope, the correlation between them, seen in Fig. 21, is unsurprising. ASASSN-15oz, having relatively steep slopes at both phases, lies at the IIL end of this relationship. This correlation was presented in Anderson et al. (2014) and is supported here with a larger sample. 


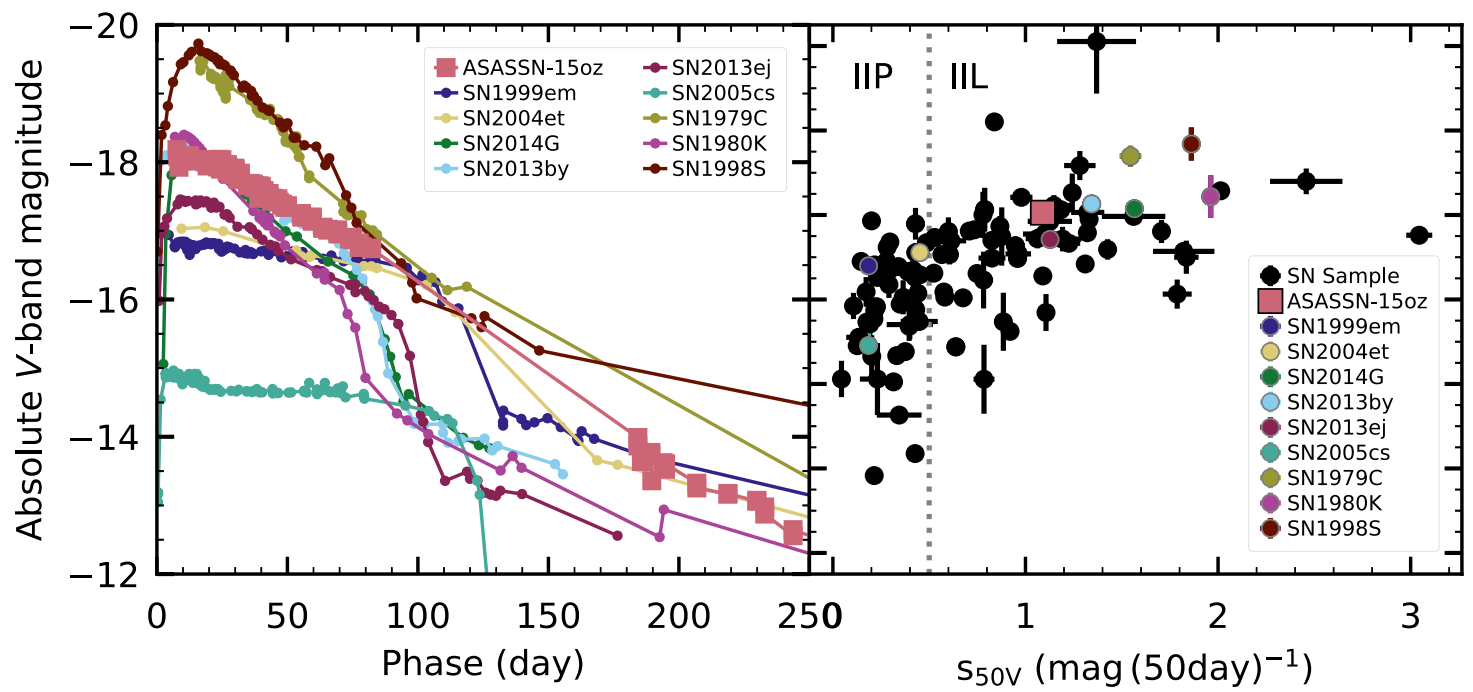

Figure 20. Left: The absolute $V$-band magnitude of ASASSN-15oz and nine well-studied SNe that span the IIP/IIL like decline rates (1979C: Balinskaia, Bychkov \& Neizvestnyi 1980; de Vaucouleurs et al. 1981; Barbon et al. 1982, 1980K: Barbon et al. 1982; Buta 1982; Tsvetkov 1983, 1998S: Fassia et al. 2000; Liu et al. 2000; Pozzo et al. 2004; Li et al. 2011, 1999em: Elmhamdi et al. 2003, 2004et: Maguire et al. 2010, 2005cs: Pastorello et al. 2009, 2013by: Valenti et al. 2015, 2013ej: Valenti et al. 2014, 2014G: Terreran et al. 2016). ASASSN-15oz is both bright and steeply declining when compared with this sample of classic SNe. Also visible in this plot is the steeper decline of the radioactive decay tail of ASASSN-15oz when compared with other objects. Right: the absolute $V$-band magnitude compared to $s_{50 \mathrm{~V}}$ for a sample of public SNe from the SNDAVIS database (black circles). The trend seen by Anderson et al. (2014) and Valenti et al. (2016) of brighter SNe to have steeper slopes is apparent. ASASSN-15oz (pink square) follows this relationship. The SNe whose light curves are shown on the left are also plotted on the right for reference. The grey dashed line denotes the separation between IIP-like and IIL-like SNe. This separation is poorly defined in the literature. The value we adopt is similar to that used by Faran et al. (2014) and Li et al. (2011).

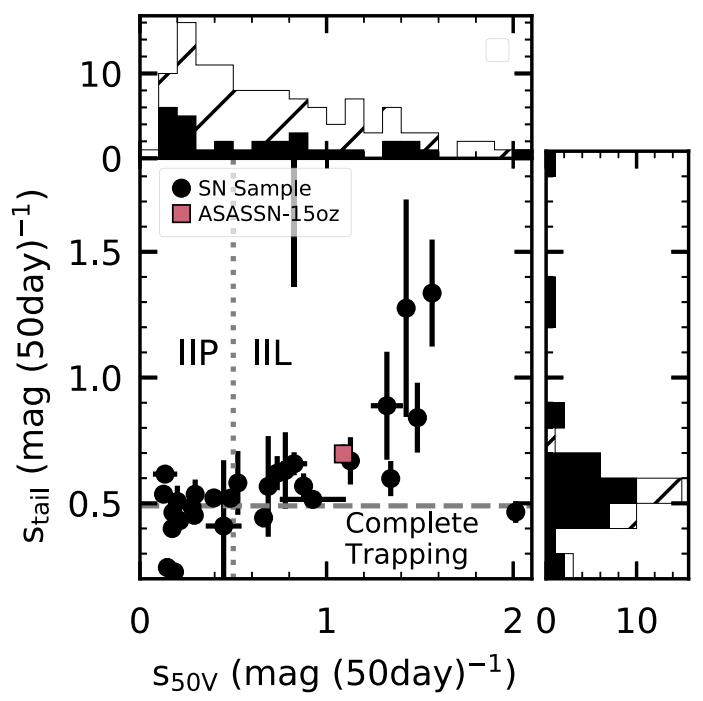

Figure 21. Centre: The slope of the light curve at late times, during the radioactive decay phase compared to $s_{50 \mathrm{v}}$ for a sample of publicly available SN light curves. ASASSN-15oz is plotted in pink. Top (Right): A histogram of the radioactive decay (photometric) phase slopes. All measured slopes from this phase are included in the hatched sample. The objects for which there is both a decay phase slope and $s_{50 \mathrm{v}}$ are plotted in black. The comparison of the full slope sample (hatch) to the combined slope sample (black) gives us confidence that points we plot in the centre are representative of the full sample. The dashed grey line marks the radioactive tail slope corresponding to complete trapping with a slope of $s_{\text {tail }}=0.5$ mag $(50 \mathrm{~d})^{-1}$. The dotted grey line represents the approximate separation between IIP and IIL SNe with a slope of $s_{50 \mathrm{v}}=0.5 \mathrm{mag}(50 \mathrm{~d})^{-1}$.
Having analysed individual observations for CSM interaction, we now turn to finding a unified model. We derived mass-loss parameters from both the light curve modelling with SNEC and the modelling of radio observations. From the optical light curve we found a compact CSM with a density of $K=4.0 \times 10^{18} \mathrm{~g} \mathrm{~cm}^{-1}$ extending to a radius of $R=1800 \mathrm{R} \odot$. Assuming a typical of RSG wind velocity between 10 and $100 \mathrm{~km} \mathrm{~s}^{-1}$, the CSM density required to fit the optical component implies a mass-loss rate between 0.8 and $8.0 \mathrm{M}_{\odot} \mathrm{yr}^{-1}$. This is significantly higher than radio mass-loss rate, $10^{-6}$ to $10^{-7} \mathrm{M}_{\odot} \mathrm{yr}^{-1}$. While the mass-loss rate from the radio observations is consistent with typical RSG mass-loss rates of $10^{-4}$ to $10^{-6} \mathrm{M}_{\odot} \mathrm{yr}^{-1}$, the optical mass-loss rate is consistent with some late stage mass-loss scenarios (e.g. Chugai 2001; Quataert \& Shiode 2012; Shiode \& Quataert 2014; Fuller 2017; Yaron et al. 2017). These discrepant values can be reconciled by considering the origin of the radiation we are observing. The excess luminosity seen in the optical light curve is due to radiation diffusion from the shocked CSM that is behind the forward shock and is only visible after shock breakout. The radio luminosity on the other hand is created by the acceleration of electrons due to the shock's interaction with the CSM. It is therefore representative of the shock's location at the time of the observation. Our observations can then be explained by a long period of average mass-loss, followed by a short period of extreme mass-loss.

With this model in mind, we examine our UV and X-ray observations, in the context of other IIP/IIL SNe. Strongly interacting SNe remain UV bright throughout their interaction, implying that perhaps a UV excess would be observed for IIP/IIL SNe during the first $30 \mathrm{~d}$, when the CSM has the greatest effect on the light curve. Furthermore, if in fact, the diversity in light curve shapes is due to mass-loss and the interaction is strong enough to produce a UV excess, then those SNe with a steeper slope, should have lost 


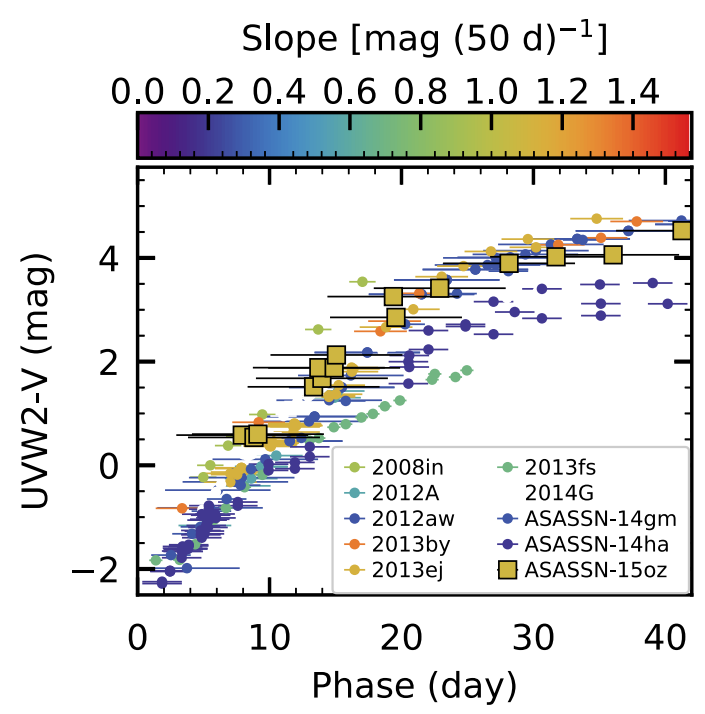

Figure 22. A comparison of the UV colour of ASASSN-15oz to that of all other IIP/IIL SNe with Swift observations and $s_{50 \mathrm{~V}}$ measurements in SNDAVIS. Each point is coloured by the $s_{50 \mathrm{~V}}$ slope, with shallower slopes in blue and steeper slopes in red. Based on this data, there is no relationship between UV excess and slope.

more mass, and therefore should be more UV bright. We explore this by plotting Swift UV colour, $U V W 2-V$, over the first $30 \mathrm{~d}$ of evolution for eight SNe in our database with Swift UVW2 and $V$-band photometry and $s_{50 \mathrm{~V}}$ measurements (Fig. 22). The symbols for each $\mathrm{SN}$ are coloured by the $V$-band slope at $50 \mathrm{~d}$. Although this sample is small, a wide range of slopes are represented and no correlation between UV excess and slope is observed. We interpret this as an indication that the CSM interaction is not strong enough to produce UV excess. However, we cannot rule out the possibility that a correlation is disrupted by uncertainties in the extinction correction.

Like the UV excess, X-rays from strongly interacting $\mathrm{SNe}$ are frequently observed. To gain more insight into the X-ray upper limit, we compare the upper limit of ASASSN-15oz with published X-ray observations of IIP/IIL SNe found in the SNaX database (Ross \& Dwarkadas 2017) (see Fig. 23). We find our upper limits are above all of the detections and upper limits of IIP/IIL SNe in the database. This further suggests that deeper observations could be needed to detect the X-ray flux of ASASSN-15oz.

\section{CONCLUSIONS}

We present extensive multiwavelength observations of the Type IIL-like SN, ASASSN-15oz, including two epochs of radio observations, four epochs of NIR spectroscopy, three epochs of NIR photometry, a $400 \mathrm{~d}$ dense multiband optical light curve, dense optical spectroscopy during the photospheric phase, three epochs of nebular optical spectroscopy, one epoch of UV spectroscopy, and $60 \mathrm{~d}$ UV and X-ray light curve.

Optical and NIR observations from the photospheric phase show ASASSN-15oz to be a typical IIL-like SN, with a linearly declining light curve and above average photospheric velocities. We find that in the explosion of ASASSN-15oz, 0.08 to $0.11 \mathrm{M}_{\odot}$ of ${ }^{56} \mathrm{Ni}$ was synthesized. However, since both the light curve modelling and the nebular spectra point towards the lower limit, we consider the lower

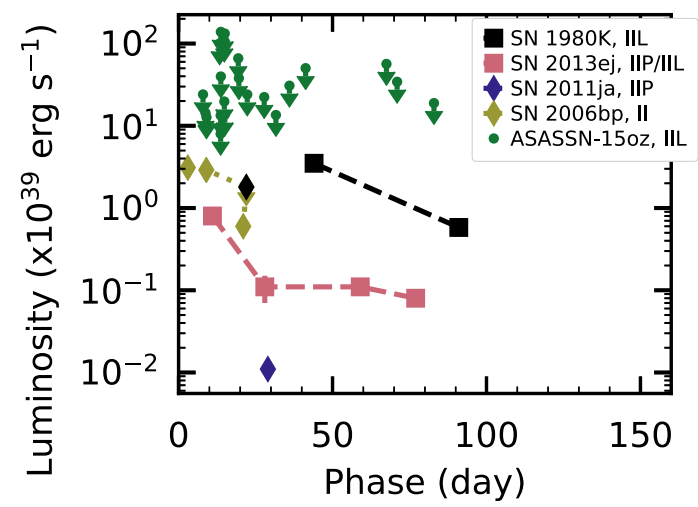

Figure 23. The X-ray luminosity of all published $\mathrm{SNe}$ found in the $\mathrm{SNaX}$ database. IIP-like SNe are shown with diamond symbols while IIL-like SNe are shown with square symbols and the upper limits as circles. All of the detections from IIP/IIL SNe are below the upper limits derived in this paper, implying that deeper observations would be required to detect its X-ray flux.

limit most likely. Its bright peak magnitude is consistent with the trend of increasing brightness with increasing photospheric slope. The steep decline of the late time light curve is also congruous with the direct correlation found between $s_{50 \mathrm{~V}}$ and radioactive tail slopes. Optical light curve modelling using SNEC finds an $17 \mathrm{M}_{\odot}$ progenitor, consistent with the range of progenitors derived from modelling of the nebular spectra.

We search for signs of interaction at all wavelengths with the following results:

(i) We detect the ASASSN-15oz in radio observations, implying a mass-loss rate of 0.9 to $9 \times 10^{-7} \mathrm{M}_{\odot} \mathrm{yr}^{-1}$ for a wind velocity between 10 and $100 \mathrm{~km} \mathrm{~s}^{-1}$.

(ii) High-velocity $\mathrm{H}$ and $\mathrm{He}$ are searched for in the NIR and optical spectra. Although there is a feature bluewards of $\mathrm{H} \alpha$, no corresponding feature is observed in any other hydrogen line nor in the He I $(\lambda 10830)$ line. For this reason we identify the cachito feature as Si II ( $\lambda 6355)$ rather than high-velocity hydrogen.

(iii) To accurately model the early optical light curve with an evolutionary progenitor, we require CSM around the RSG progenitor with a density of $4 \times 10^{18} \mathrm{~g} \mathrm{~cm}^{-1}$ and radial extent of $1800 \mathrm{R}_{\odot}$, implying a short period of extreme mass-loss.

(iv) No narrow emission lines are detected in the optical and UV photospheric spectroscopy, implying a mass-loss rate of $<10^{-4}$ $\mathrm{M}_{\odot} \mathrm{yr}^{-1}$.

(v) Although the $\mathrm{H} \alpha$ emission line profile in the nebular spectra is asymmetric, we do not interpret this as interaction with CSM.

(vi) No UV excess is detected in the first $40 \mathrm{~d}$ of observations.

(vii) No X-ray signal is detected in any of the photospheric observations.

Taking all of these results together, we find the progenitor of ASASSN-15oz underwent standard RSG mass-loss for most of its evolution, with a short period of extreme mass-loss just before explosion. It is possible that early optical spectroscopy would have shown narrow emission lines as the shock illuminated the edge of the dense shell of material. Additionally, an X-ray detection may have been made with deeper observations. A dense shell of material surrounded by a lower density CSM has now been used to model the observations of a handful of objects (e.g. SN 1998S: Chugai 
2001; SN 2013fs: Yaron et al. 2017; SN 2013ej: Morozova \& Stone 2018). As the best model to explain the observations of an IIP-like SN (SN 2013fs), a transition SN (SN 2013ej), and now to an IILlike SN (ASASSN-15oz) it may be applicable to a broad range of progenitors.

\section{ACKNOWLEGMENTS}

All scripted parts of this analysis, including the paper can be found at https://github.com/abostroem/asassn15oz.

We would like to thank Dr Nikolai Chugai for his insightful comments.

This work was supported by the Swift Guest Observers Program through grant NNX16AE90G. This work is based (in part) on observations collected at the European Organization for Astronomical Research in the Southern Hemisphere, Chile as part of PESSTO [the Public European Southern Observatory (ESO) Spectroscopic Survey for Transient Objects Survey], ESO program 188.D-3003, 191.D-0935, 197.D-1075. Based on observations collected at the European Organization for Astronomical Research in the Southern Hemisphere under ESO program 095.A-0316(A). We acknowledge support from EU/FP7-ERC (European Research Council) grant [615929]. DAH, GH, and CM are supported by National Science Foundation (NSF) grant AST-1313484. KML acknowledges funding from the European Research Council under ERC Consolidator Grant agreement no. 647208. ALP acknowledges financial support for this research from a Scialog award made by the Research Corporation for Science Advancement. OR acknowledges support by projects IC120009 'Millennium Institute of Astrophysics (MAS)' of the Iniciativa Científica Milenio del Ministerio Economía, Fomento y Turismo de Chile, and CONICYT PAI/INDUSTRIA 79090016. DJS is a visiting astronomer at the Infrared Telescope Facility, which is operated by the University of Hawaii under contract NNH14CK55B with the National Aeronautics and Space Administration. Research by DJS is supported by NSF grants AST-1821967, 1821987, 1813708, and 1813466.

This research has made use of the NASA/IPAC Extragalactic Database (NED), which is operated by the Jet Propulsion Laboratory, California Institute of Technology, under contract with the National Aeronautics and Space Administration. This work uses the National Radio Astronomy Observatory which is a facility of the National Science Foundation operated under cooperative agreement by Associated Universities, Inc. This work makes use of observations from the Las Cumbres Observatory network. This work was partly supported by the United Kingdom Space Agency. Based on observations obtained at the Gemini Observatory through proposal GS-2016A-Q-75-25, acquired through the Gemini Observatory Archive, and processed using the GEMINI IRAF package, which is operated by the Association of Universities for Research in Astronomy, Inc., under a cooperative agreement with the NSF on behalf of the Gemini partnership: the National Science Foundation (United States), the National Research Council (Canada), CONICYT (Chile), Ministerio de Ciencia, Tecnología e Innovación Productiva (Argentina), and Ministério da Ciência, Tecnologia e Inovação (Brazil).

This research made use of ASTROPY,${ }^{10}$ a community-developed core PYTHON package for Astronomy (Astropy Collaboration 2013; Price-Whelan et al. 2018). Support for GP is provided by the Ministry of Economy, Development, and Tourism's Millennium Science

\footnotetext{
${ }^{10} \mathrm{http}: / /$ www.astropy.org
}

Initiative through grant IC120009, awarded to The Millennium Institute of Astrophysics, MAS.

\section{REFERENCES}

Anderson J. P. et al., 2014, ApJ, 786, 67

Arcavi I. et al., 2012, ApJ, 756, L30

Astropy Collaboration et al., 2013, aap, 558, A33

Balinskaia I. S., Bychkov K. V., Neizvestnyi S. I., 1980, A\&A, 85, L19

Barbon R., Ciatti F., Rosino L., 1979, A\&A, 72, 287

Barbon R., Ciatti F., Rosino L., Ortolani S., Rafanelli P., 1982, A\&A, 116, 43

Blinnikov S. I., Bartunov O. S., 1993, A\&A, 273, 106

Blondin S., Tonry J. L., 2007, ApJ, 666, 1024

Blondin S., Tonry J. L., 2011, Astrophysics Source Code Library, record ascl:1107.001

Breeveld A. A. et al., 2010, MNRAS, 406, 1687

Brown P. J. et al., 2009, AJ, 137, 4517

Brown T. M. et al., 2013, PASP, 125, 1031

Brown J. S. et al., 2015, Astron. Telegram, 7989, 1

Bullivant C. et al., 2018, MNRAS, 476, 1497

Buta R. J., 1982, PASP, 94, 578

Buzzoni B. et al., 1984, The Messenger, 38, 9

Cardelli J. A., Clayton G. C., Mathis J. S., 1989, ApJ, 345, 245

Chevalier R. A., 1982, ApJ, 259, 302

Chevalier R. A., 1998, ApJ, 499, 810

Chevalier R. A., Fransson C., 2006, ApJ, 651, 381

Childress M. J. et al., 2016, Publ. Astron. Soc. Aust., 33, e055

Chugai N. N., 1992, SvA, 36, 63

Chugai N. N., 2001, MNRAS, 326, 1448

Chugai N. N., Fabrika S. N., Sholukhova O. N., Goranskij V. P., Abolmasov P. K., Vlasyuk V. V., 2005, Astron. Lett., 31, 792

Chugai N. N., Chevalier R. A., Utrobin V. P., 2007, ApJ, 662, 1136

Cushing M. C., Vacca W. D., Rayner J. T., 2004, PASP, 116, 362

Davies B., Beasor E. R., 2018, MNRAS, 474, 2116

de Vaucouleurs G., de Vaucouleurs A., Buta R., Ables H. D., Hewitt A. V., 1981, PASP, 93, 36

Dessart L., Hillier D. J., 2005, A\&A, 437, 667

Dhungana G. et al., 2016, ApJ, 822, 6

Dwarkadas V. V., 2014, MNRAS, 440, 1917

Eastman R. G., Schmidt B. P., Kirshner R., 1996, ApJ, 466, 911

Elmhamdi A. et al., 2003, MNRAS, 338, 939

Falk S. W., Arnett W. D., 1977, ApJS, 33, 515

Faran T. et al., 2014, MNRAS, 442, 844

Fassia A. et al., 2000, MNRAS, 318, 1093

Ferguson J. W., Alexander D. R., Allard F., Barman T., Bodnarik J. G., Hauschildt P. H., Heffner-Wong A., Tamanai A., 2005, ApJ, 623, 585

Förster F. et al., 2018, Nat. Astron., 2, 808

Freudling W., Romaniello M., Bramich D. M., Ballester P., Forchi V., GarcíaDabló C. E., Moehler S., Neeser M. J., 2013, A\&A, 559, A96

Fuller J., 2017, MNRAS, 470, 1642

Gal-Yam A. et al., 2014, Nature, 509, 471

Galbany L. et al., 2016, AJ, 151, 33

Gezari S. et al., 2015, ApJ, 804, 28

Grassberg E. K., Imshennik V. S., Nadyozhin D. K., 1971, Ap\&SS, 10, 28

Gutiérrez C. P. et al., 2014, ApJ, 786, L15

Gutiérrez C. P. et al., 2017, ApJ, 850, 89

Gutiérrez C. P. et al., 2018, MNRAS, 479, 3232

Hamuy M. A., 2001, PhD thesis, The University of Arizona

Heger A., Fryer C. L., Woosley S. E., Langer N., Hartmann D. H., 2003, ApJ, 591, 288

Hook I. M., Jørgensen I., Allington-Smith J. R., Davies R. L., Metcalfe N., Murowinski R. G., Crampton D., 2004, PASP, 116, 425

Horesh A. et al., 2013, MNRAS, 436, 1258 
Hosseinzadeh G., Valenti S., Arcavi I., McCully C., Howell D. A., 2015, Astron. Telegram, 7997, 1

Iglesias C. A., Rogers F. J., 1996, ApJ, 464, 943

Inserra C. et al., 2013, A\&A, 555, A142

Jerkstrand A., Fransson C., Kozma C., 2011, A\&A, 530, A45

Jerkstrand A., Fransson C., Maguire K., Smartt S., Ergon M., Spyromilio J., 2012, A\&A, 546, A28

Jerkstrand A., Smartt S. J., Fraser M., Fransson C., Sollerman J., Taddia F., Kotak R., 2014, MNRAS, 439, 3694

Jerkstrand A., Ertl T., Janka H. T., Müller E., Sukhbold T., Woosley S. E., 2018, MNRAS, 475, 277

Kalberla P. M. W., Burton W. B., Hartmann D., Arnal E. M., Bajaja E., Morras R., Pöppel W. G. L., 2005, A\&A, 440, 775

Kasen D., Woosley S. E., 2009, ApJ, 703, 2205

Kiewe M. et al., 2012, ApJ, 744, 10

Kochanek C. S., 2019, MNRAS, 483, 3762

Kuin P., 2014, Astrophysics Source Code Library, record ascl:1410.004

Kuin N. P. M. et al., 2015, MNRAS, 449, 2514

Leonard D. C. et al., 2002, PASP, 114, 35

Li W. et al., 2011, MNRAS, 412, 1441

Liu Q. Z., Hu J. Y., Hang H. R., Qiu Y. L., Zhu Z. X., Qiao Q. Y., 2000, A\&AS, 144, 219

Maguire K. et al., 2010, MNRAS, 404, 981

Mauron N., Josselin E., 2011, A\&A, 526, A156

Minkowski R., 1941, PASP, 53, 224

Minkowski R., 1964, ARA\&A, 2, 247

Moriya T. J., Yoon S.-C., Gräfener G., Blinnikov S. I., 2017, MNRAS, 469, L108

Morozova V., Stone J. M., 2018, ApJ, 867, 4

Morozova V., Piro A. L., Renzo M., Ott C. D., Clausen D., Couch S. M., Ellis J., Roberts L. F., 2015, ApJ, 814, 63

Morozova V., Piro A. L., Valenti S., 2017, ApJ, 838, 28

Morozova V., Piro A. L., Valenti S., 2018, ApJ, 858, 15

Mukai K., 1993, Legacy, 3, 21

Nadyozhin D. K., 1985, Ap\&SS, 112, 225

Nieuwenhuijzen H., de Jager C., 1990, A\&A, 231, 134

Paczynski B., 1983, ApJ, 267, 315

Pastorello A. et al., 2009, MNRAS, 394, 2266

Patat F., Barbon R., Cappellaro E., Turatto M., 1993, A\&AS, 98, 443

Patat F., Barbon R., Cappellaro E., Turatto M., 1994, A\&A, 282, 731

Paxton B. et al., 2018, ApJS, 234, 34

Poznanski D., Prochaska J. X., Bloom J. S., 2012, MNRAS, 426, 1465

Pozzo M., Meikle W. P. S., Fassia A., Geballe T., Lundqvist P., Chugai N. N., Sollerman J., 2004, MNRAS, 352, 457

Price-Whelan A. M. et al., 2018, AJ, 156, 123

Pskovskii Y. P., 1967, SvA, 11, 63

Quataert E., Shiode J., 2012, MNRAS, 423, L92

Rayner J. T., Toomey D. W., Onaka P. M., Denault A. J., Stahlberger W. E.,

Vacca W. D., Cushing M. C., Wang S., 2003, PASP, 115, 362

Ross M., Dwarkadas V. V., 2017, AJ, 153, 246

Rubin A., Gal-Yam A., 2016, ApJ, 828, 111

Sanders N. E. et al., 2015, ApJ, 799, 208

Savitzky A., Golay M. J. E., 1964, Anal. Chem., 36, 1627

Schlafly E. F., Finkbeiner D. P., 2011, ApJ, 737, 103

Schlegel E. M., 1996, AJ, 111, 1660

Shiode J. H., Quataert E., 2014, ApJ, 780, 96
Silverman J. M., Kong J. J., Filippenko A. V., 2012, MNRAS, 425, 1819

Smartt S. J., 2015, Publ. Astron. Soc. Aust., 32, e016

Smartt S. J. et al., 2015, A\&A, 579, A40

Smitka M. T., 2015, Astrophysics Source Code Library, record ascl:1509.005

Soderberg A. M. et al., 2012, ApJ, 752, 78

Spiro S. et al., 2014, MNRAS, 439, 2873

Stetson P. B., 1987, PASP, 99, 191

Sukhbold T., Woosley S. E., 2014, ApJ, 783, 10

Sukhbold T., Ertl T., Woosley S. E., Brown J. M., Janka H.-T., 2016, ApJ, 821,38

Terreran G. et al., 2016, MNRAS, 462, 137

Thomas R. C., 2013, Astrophysics Source Code Library, record ascl:1308.008

Thomas R. C., Nugent P. E., Meza J. C., 2011, PASP, 123, 237

Tomasella L. et al., 2013, MNRAS, 434, 1636

Tomasella L. et al., 2018, MNRAS, 475, 1937

Tsvetkov D. Y., 1983, Perem. Zvezdy, 22, 39

Utrobin V. P., Chugai N. N., 2008, A\&A, 491, 507

Utrobin V. P., Chugai N. N., 2017, MNRAS, 472, 5004

Vacca W. D., Cushing M. C., Rayner J. T., 2003, PASP, 115, 389

Valenti S. et al., 2008, MNRAS, 383, 1485

Valenti S. et al., 2011, MNRAS, 416, 3138

Valenti S. et al., 2014, MNRAS, 438, L101

Valenti S. et al., 2015, MNRAS, 448, 2608

Valenti S. et al., 2016, MNRAS, 459, 3939

Vernet J. et al., 2011, A\&A, 536, A105

Walmswell J. J., Eldridge J. J., 2012, MNRAS, 419, 2054

Weaver T. A., Zimmerman G. B., Woosley S. E., 1978, ApJ, 225, 1021

Weiler K. W., Panagia N., Montes M. J., Sramek R. A., 2002, ARA\&A, 40, 387

Wellstein S., Langer N., 1999, A\&A, 350, 148

Woosley S. E., Heger A., 2007, Phys. Rep., 442, 269

Woosley S. E., Heger A., 2015, ApJ, 810, 34

Woosley S. E., Weaver T. A., 1995, ApJS, 101, 181

Yaron O. et al., 2017, Nat. Phys., 13, 510

Yaron O., Gal-Yam A., 2012, PASP, 124, 668

Young T. R., Branch D., 1989, ApJ, 342, L79

Zaghloul M. R., Bourham M. A., Doster J. M., 2000, J. Phys. D: Appl. Phys., 33, 977

\section{SUPPORTING INFORMATION}

Supplementary data are available at MNRAS online.

A complete table of photometric observations is available as a comma-separated value file in the supplementary data. Table A2 shows the first five rows of this supplemental table.

Please note: Oxford University Press is not responsible for the content or functionality of any supporting materials supplied by the authors. Any queries (other than missing material) should be directed to the corresponding author for the article.

\section{APPENDIX A: TABLES OF OBSERVATIONS}


Table A1. Spectroscopic observations of ASASSN-15oz.

\begin{tabular}{lcccc}
\hline Date & JD & Phase (d) & Observatory & Instrument \\
\hline $2015-09-04$ & 2457270.0 & 8.0 & LCO & FLOYDS \\
$2015-09-05$ & 2457270.7 & 8.7 & Swift & UVOTA \\
$2015-09-05$ & 2457271.1 & 9.1 & Swift & UVOTA \\
$2015-09-05$ & 2457270.6 & 8.6 & NTT & SOFI \\
$2015-09-06$ & 2457272.0 & 10.0 & LCO & FLOYDS \\
$2015-09-07$ & 2457273.0 & 11.0 & LCO & FLOYDS \\
$2015-09-10$ & 2457275.7 & 13.7 & Swift & UVOTA \\
$2015-09-10$ & 2457275.7 & 13.7 & Swift & UVOTA \\
$2015-09-11$ & 2457277.0 & 15.0 & LCO & FLOYDS \\
$2015-09-11$ & 2457276.9 & 14.9 & Swift & UVOTA \\
$2015-09-16$ & 2457282.0 & 20.0 & LCO & FLOYDS \\
$2015-09-20$ & 2457286.1 & 24.1 & LCO & FLOYDS \\
$2015-09-21$ & 2457287.5 & 25.5 & VLT & X-SHOOTER \\
$2015-09-24$ & 2457290.0 & 28.0 & LCO & FLOYDS \\
$2015-09-30$ & 2457296.0 & 34.0 & LCO & FLOYDS \\
$2015-10-04$ & 2457299.5 & 37.5 & NTT & EFOSC \\
$2015-10-05$ & 2457300.5 & 38.5 & NTT & SOFI \\
$2015-10-06$ & 2457301.9 & 39.9 & LCO & FLOYDS \\
$2015-10-10$ & 2457305.7 & 43.7 & IRTF & SpeX \\
$2015-10-14$ & 2457310.0 & 48.0 & LCO & FLOYDS \\
$2015-10-25$ & 2457320.9 & 58.9 & LCO & FLOYDS \\
$2015-11-07$ & 2457333.9 & 71.9 & LCO & FLOYDS \\
$2015-11-08$ & 2457334.5 & 72.5 & NTT & EFOSC \\
$2015-11-19$ & 2457345.5 & 83.5 & NTT & EFOSC \\
$2016-04-11$ & 2457489.9 & 227.9 & NTT & EFOSC \\
$2016-04-11$ & 2457489.9 & 227.9 & NTT & EFOSC \\
$2016-06-09$ & 2457548.8 & 286.8 & Gemini-S & GMOS \\
$2016-06-10$ & 2457549.7 & 287.7 & Gemini-S & GMOS \\
$2016-06-12$ & 2457551.7 & 289.7 & Gemini-S & GMOS \\
$2016-08-03$ & 2457603.7 & 341.7 & NTT & EFOSC \\
$2016-09-11^{a}$ & 2457642.6 & 380.6 & NTT & EFOSC \\
$2016-09-11^{a}$ & 2457642.6 & 380.6 & NTT & EFOSC \\
$2016-09-19$ & 2457650.5 & 388.5 & NTT & EFOSC \\
$2017-09-20^{b}$ & 2458016.9 & 754.9 & Swift & UVOTA \\
\hline No & $249-1 i n$ & & &
\end{tabular}

${ }^{a}$ No signal in data due to cloud cover.

${ }^{\mathrm{b}}$ Template observation.

Table A2. Sample of imaging observations of ASASSN-15oz. Full table available online.

\begin{tabular}{lcccccc}
\hline Date-Obs & JD & Phase (d) & Apparent magnitude & Apparent magnitude error & Filter & Source \\
\hline $2015-09-04$ & 2457269.68 & 7.68 & 14.64 & 0.02 & $B$ & LSC $1 \mathrm{~m}$ \\
$2015-09-04$ & 2457269.68 & 7.68 & 14.66 & 0.02 & B & LSC $1 \mathrm{~m}$ \\
$2015-09-04$ & 2457269.69 & 7.69 & 14.37 & 0.02 & $V$ & LSC $1 \mathrm{~m}$ \\
$2015-09-04$ & 2457269.69 & 7.69 & 14.43 & 0.01 & $g$ & LSC $1 \mathrm{~m}$ \\
$2015-09-04$ & 2457269.69 & 7.69 & 14.42 & 0.01 & $g$ & LSC $1 \mathrm{~m}$ \\
\hline
\end{tabular}

\section{APPENDIX B: LINE IDENTIFICATION AND FITTING DETAILS}

For lines that are blended (but still showed partially isolated absorption troughs) we define the continuum as a straight line from the lowest side of the feature. After dividing by the continuum, we then simultaneously fit multiple 1D Gaussian profiles to the continuum normalized spectra. For blended features originating in the same part of the ejecta, we require that each component has the same width. We define the velocity of a feature as the minimum (mean) of the individual Gaussian profiles corresponding to that ion and the error as the standard deviation of the fit, representing the range of velocities present in the photosphere. An example of the multiple components and the combined fit is shown in the left-hand panel of Fig. B1. For the Ca II NIR triplet we use the additional constraint of a fixed offset between the minima of the features.

For unblended lines, we perform the following steps to find the minimum and the standard deviation (following Silverman et al. 2012). We eliminate large noise spikes (e.g. from cosmic rays) using a Savitzky-Golay smoothing filter (Savitzky \& Golay 1964) with a quadratic function over a binsize of five pixels. For each line, a minimum and maximum wavelength is defined as well as a slope to account for the shifting of the feature over time as the ejecta slows. A slope is then fit to small bins over this wavelength 
range, starting with a binsize of five pixels and increasing until exactly three changes in slope are found (at either edge of the line and the line centre). If the binsize reaches 40 per cent of the feature size, no further attempt at fitting is made. The feature edges are confirmed by fitting a quadratic function to the region centred on each edge identified by the slope change with a width of 20 pixels for the FLOYDS spectra and five pixels for the EFOSC2 spectra. The edge is considered successfully found if the quadratic fit is concave down. The continuum is defined by a line though these end points. Finally, the velocity is found by fitting a cubic spline to the continuum subtracted flux between the edges using the flux errors as weights. An example of a feature with the continuum and spline fit is shown in the right-hand panel of Fig. B1.

We use both methods to fit $\mathrm{NaI}$ ( $\lambda$ 5898) and $\mathrm{H} \beta$ ( $\lambda$ 4861). For $\mathrm{Na}$, we find the spline fit does not characterize the minimum of the profile well and instead use the Gaussian minimum. The $\mathrm{H} \beta$ profile is contaminated by another feature (possibly Ti II) at later times, offsetting the minimum found using the spline fit. For this reason we prefer the Gaussian fit for $\mathrm{H} \beta$ as well. A comparison of the results of both fits for $\mathrm{H} \beta$ at early times finds them in good agreement, giving us confidence in the consistency of the two methods.

Table B1. The best parameters for the SYN ++ fit. The Sobolev optical depth $\tau$ is modelled with an exponential profile with e-folding length, aux, minimum velocity, $v_{\min }$, and maximum velocity, $v_{\max }$. The temperature column is the Boltzmann excitation temperature. SYN ++ models pure resonance scattering which is a poor approximation for the $\mathrm{H} \alpha$. For this reason we use the $\mathrm{H} \beta$ line to determine the hydrogen contribution to the fit. A separate fit is performed for $\mathrm{H}$ $\alpha$ and is listed separately in the table. We emphasize that this fit is used for line identification and not to derive ejecta properties.

\begin{tabular}{lccccc}
\hline Ion & $\log (\tau)$ & $v_{\min }\left(\mathrm{kkm} \mathrm{s}^{-1}\right)$ & $v_{\max }\left(\mathrm{kkm} \mathrm{s}^{-1}\right)$ & aux & Temperature $(\mathrm{kK})$ \\
\hline H I $\alpha$ & 0.06 & 0.1 & 40.0 & 2.0 & 10.0 \\
H I $\beta$ & 1.1 & 0.1 & 40.0 & 2.0 & 10.0 \\
Na II & -0.5 & 0.1 & 40.0 & 1.0 & 10.0 \\
O I & -0.8 & 0.1 & 40.0 & 1.0 & 10.0 \\
Ca II & 1.3 & 0.1 & 40.0 & 2.0 & 10.0 \\
Sc II & 0.1 & 0.1 & 40.0 & 1.0 & 10.0 \\
Ti II & 0.3 & 0.1 & 40.0 & 1.0 & 10.0 \\
Fe I & 0.3 & 0.1 & 40.0 & 1.0 & 10.0 \\
Fe II & 0.3 & 0.1 & 40.0 & 1.0 & 7.0 \\
Ba I & 0.0 & 0.1 & 40.0 & 1.0 & 10.0 \\
\hline
\end{tabular}
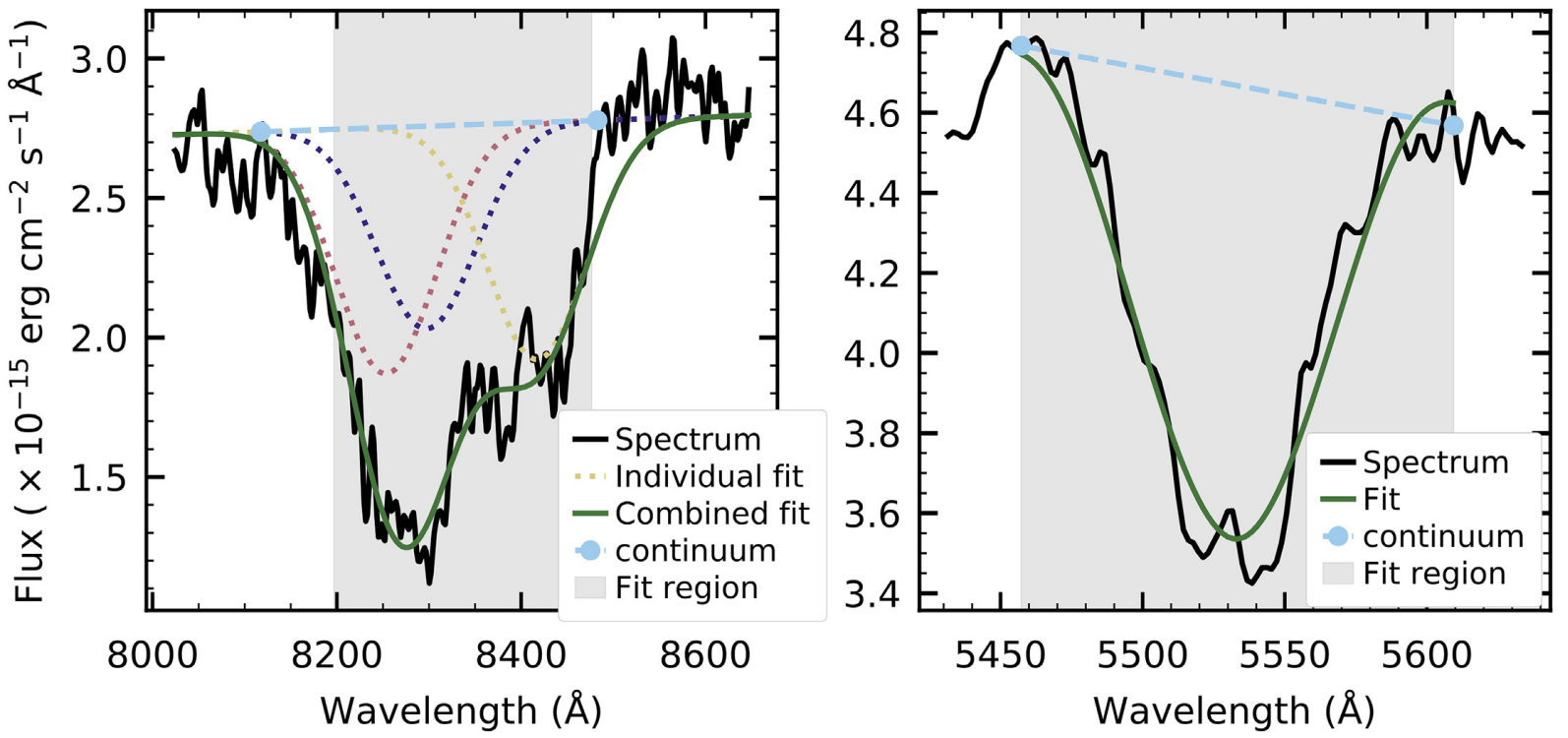

Figure B1. An example of a fit to the multicomponent Ca II NIR triplet (left) using multiple Gaussians and a single line fit (following Silverman et al. 2012, right) to the Sc II ( $\lambda$ 5662) line. The observed spectrum is plotted in black. The continuum edges are marked with a cyan circle and the cyan dashed line connecting these points is used as the continuum. The Ca II NIR triplet fit is found by simultaneously fitting three Gaussians with the same standard deviation and mean offsets corresponding to the expected wavelength separation of the triplet. The individual Gaussians are plotting at as dotted lines and the combined fit is plotted as a solid green line. The minima of the individual Gaussians is used to find the velocity of each component. The Sc II feature is fit with a cubic spline. The minimum of the spline is used to find the Sc II velocity. 
${ }^{1}$ Department of Physics, University of California, Davis, CA 95616, USA

${ }^{2}$ Racah Institute of Physics, The Hebrew University of Jerusalem, Jerusalem 91904, Israel

${ }^{3}$ Department of Astrophysical Sciences, Princeton University, Princeton, $N J$ 08544, USA

${ }^{4}$ Mullard Space Science Laboratory, University College London, Holmbury St. Mary, Dorking, Surrey RH5 6NT, UK

${ }^{5}$ Department of Astronomy/Steward Observatory, 933 North Cherry Avenue, Rm. N204, Tucson, AZ 85721-0065, USA

${ }^{6}$ Max-Planck Institut für Astrophysik, Karl-Schwarzschild Str 1, D-85748 Garching, Germany

${ }^{7}$ School of Physics and Astronomy, University of Southampton, Southampton SO17 1BJ, UK

${ }^{8}$ Harvard-Smithsonian Center for Astrophysics, 60 Garden Street, Cambridge, MA 02138, UK

${ }^{9}$ The Raymond and Beverly Sackler School of Physics and Astronomy, Tel Aviv University, Tel Aviv 69978, Israel

${ }^{10}$ School of Physics, O'Brien Centre for Science North, University College Dublin, Belfield, Dublin 4, Ireland

${ }^{11}$ Cerro Tololo Inter-American Observatory, National Optical Astronomy Observatory, Casilla 603, La Serena, Chile

${ }^{12}$ Department of Particle Physics and Astrophysics, Weizmann Institute of Science, Rehovot 7610001, Israel

${ }^{13}$ PITT PACC, Department of Physics and Astronomy, University of Pittsburgh, Pittsburgh, PA 15260, USA
${ }^{14}$ Department of Physics, University of California, Santa Barbara, CA 93106-9530, USA

${ }^{15}$ Las Cumbres Observatory, 6740 Cortona Dr Ste 102, Goleta, CA 931175575, USA

${ }^{16}$ School of Physics \& Astronomy, Cardiff University, Queens Buildings, The Parade, Cardiff CF24 3AA, UK

${ }^{17}$ Astrophysics Research Centre, School of Mathematics and Physics, Queens University Belfast, Belfast BT7 1NN, UK

${ }^{18}$ Tuorla Observatory, Department of Physics and Astronomy, University of Turku, FI-20014 Turku, Finland

${ }^{19}$ SRON Netherlands Institute for Space Research, Sorbonnelaan 2, NL3584 CA Utrecht, the Netherlands

${ }^{20}$ Department of Astrophysics/IMAPP, Radboud University, P.O. Box 9010, NL-6500 GL Nijmegen, the Netherlands

${ }^{21}$ Departamento de Ciencias Fisicas, Universidad Andres Bello, Avda. Republica 252, Santiago, Chile

${ }^{22}$ Millennium Institute of Astrophysics (MAS), Nuncio Monseñor Sótero Sanz 100, Providencia, Santiago, Chile

${ }^{23}$ The Observatories of the Carnegie Institution for Science, 813 Santa Barbara Street, Pasadena, CA 91101, USA

This paper has been typeset from a $\mathrm{T}_{\mathrm{E}} \mathrm{X} / \mathrm{LT} \mathrm{T} \mathrm{X}$ file prepared by the author. 